\title{
How do H-bonding interactions control viscoelasticity and thixotropy of molecular gels? Insights from mono-, di- and tri-hydroxymethylated alkanamide gelators.
}

\author{
Yan Zhang $^{\mathrm{a}, \mathrm{b}}$ and Richard G. Weiss* ${ }^{\mathrm{b}, \mathrm{c}}$ \\ a Graduate School, Changchun University of Technology (CCUT), 130012, \\ Changchun, P. R. China. \\ ${ }^{\mathrm{b}}$ Department of Chemistry and ${ }^{\mathrm{c}}$ Institute for Soft Matter Synthesis and Metrology, \\ Georgetown University, Washington, DC 20057-1227, USA. E-mail: \\ weissr@georgetown.edu.
}

\begin{abstract}
The structural and dynamic properties of molecular gels, made from 9 structurally-related mono-, di, and tri-hydroxymethylated alkanamide gelators, have been examined at different distance scales. The subtle changes in the gelator structures, in terms of the number of hydroxymethyl groups and the length of the alkanamide chain, have been correlated with the type of the self-assembled fibrillar networks and the viscoelasticity of the gels as well as the characteristics of the liquid as indicated by Hansen solubility parameters.
\end{abstract}

Some of the gels exhibit high degrees of thixotropy and very rapid recovery after the cessation of destructive strain. Gelation efficiencies-based upon the range of liquids gelated, the critical gelator concentrations, and the gel-sol transition temperatures - depend upon both the length of the fatty acid chain and the number of hydroxymethyl groups: the best gelator of the series contains the longest alkyl chain examined (hexadecyl) and two hydroxymethyl groups (i.e., better than the gelators with one or three groups). FT-IR and powder X-ray diffraction data indicate that hydrogen-bonding and molecular packing modes in the gels and the neat gelator powders are very similar. Polarizing optical microscopy images of the gels show that 
the morphology of the gel networks can be tuned by changing the cooling processes used to transform the sols into gels. In total, the observations and conclusions derived provide useful insights into the relationship between gelator structure and gel properties. These data will be useful to those interested in the a priori design of new gelators or other molecules undergoing a variety of self-assembly processes that lead to robust thermal- and mechano-reversible materials.

\section{INTRODUCTION}

Molecular gels are a type of soft matter comprised of a low concentration of a low-molecular-weight gelator (LMOG) and a liquid. The gel phases are formed through aggregation and nucleation of the LMOG molecules into self-assembled, three-dimensional networks (SAFINs) that immobilize the liquid component. ${ }^{1-4}$ The driving forces for the self-assembly can include non-covalent interactions like H-bonding, $\pi-\pi$ stacking, donor-acceptor interactions and van der Waals interactions. $^{5-8}$

Several properties of molecular gels make them attractive candidates for applications in the cosmetics, ${ }^{9}$ drug delivery, ${ }^{10}$ and food industries. ${ }^{11}$ Those properties include thermal reversibility - they can be cycled between solutions/sols and gels phases by heating above and cooling below a characteristic temperature, $T_{g e l}$-and sensitivity to external stimuli, such as mechanical stress that leads to their reversion to sol phases. However, some molecular gels recover part or nearly all of their viscoelasticity after cessation of destructive mechanical stress (i.e., they are partially or fully thixotropic). Thixotropic materials are useful in the food industry, to mitigate fracture and fatigue, and as bio- and self-healing materials. ${ }^{12-14}$ Many of the known thixotropic molecular gels rely upon LMOGs that contain a metal ion, ${ }^{15,16}$ derivatives of steroids (especially cholesterol ${ }^{17,18}$ ), ${ }^{19,20}$ porphyrins, $^{21}$ dianthracenes, ${ }^{22}$ simple amides, $^{23}$ and ureas. ${ }^{24}$

A structurally simple LMOG, N-3-hydroxypropyl dodecanamide, has been reported to produce thixotropic gels with toluene as the liquid. ${ }^{23}$ In that case, the 
thixotropic behavior was induced by shear-induced alignment of rod-like objects and reformation of the connections among them after the cessation of shear. The degree of viscoelastic recovery of gels with cholesterol derivatives as LMOGs is influenced greatly by the morphology of the SAFINs: spherulitic aggregates, consisting of highly branched fibers, appear to favor recovery much more than networks consisting of rod-like objects. ${ }^{25}$ More recently, we have studied the relationship between the molecular structures of amide and amine LMOGs based on $(R)$-12-hydroxystearic acid (12HSA) and their gelating abilities and thixotropic behaviors in organic liquids. $^{26,27}$ The results indicate that the degree of viscoelastic recovery can be correlated qualitatively with the strength of hydrogen-bonding interactions among the LMOG molecules. Also, an interesting concentration dependence on thixotropic behavior has been reported for toluene gels prepared with some asymmetric peptide-based compounds: ${ }^{28}$ within a concentration range of gelator, the gels exhibited thixotropic properties while those formed either below or above that range did not.

Here, the gelation efficiencies and thixotropic properties of molecular gels based upon a series of structurally simple gelators, m-HMMnA (Figure 1, where $\mathrm{m}$ is the number of hydroxymethyl groups attached to the carbon atom adjacent to nitrogen and $\mathrm{n}$ is the length of an $n$-alkyl chain connected to an amide group), have been explored. The selection of hydroxymethyl groups is based upon their appearance in several natural products with physiological activity and drugs derived from them ${ }^{29-31}$ and their use for the controlled release of drugs, ${ }^{32}$ as well as the ability to explore in detail the effect of increasing or decreasing H-bonding interactions in selective parts of the gelator structures. ${ }^{33-36}$ Previous studies with molecules of greater structural complexity have demonstrated that the placement and number of hydroxyl groups can have important consequences to how self-assembly occurs. ${ }^{37-41}$ In addition, the ability to explore the balance among the influences to m-HMMnA self-assembly from H-bonding, van der Waals forces (through changing the length of the alkyl chains), and secondary amide group intermolecular interactions are built into the m-HMMnA 
structures. In that regard, the gelator efficiencies of the m-HMMnA have been correlated with structural analyses of the gel networks at different distance scales in order to discern the salient factors needed to produce gels with specific properties. Somewhat surprisingly, we find that the degree of viscoelastic recovery is not very sensitive to the amount of destructive strain applied to the gels; in essence, the SAFINs are being disrupted but not totally destroyed. ${ }^{27,42}$ However, the gel properties are very sensitive to the number of hydroxymethyl groups, the length of the alkanamide chain, the LMOG concentration, and the properties of the liquids as assessed by Hansen solubility parameters. ${ }^{43-45}$ Contrary to our initial expectations, the 3-HMMnA series of gelators did not produce the strongest gels, and reasons for this observation are advanced based on the competition between intra- and inter-molecular H-bonding interactions within the SAFIN assemblies. We anticipate that even more robust thermal- and mechano-reversible molecular gels and other related self-assembling systems can be produced by judicious use of the information presented here.<smiles>O=C(CCCCCCCCO)NCCO</smiles>

1-HMMnA<smiles>O=C(CCCCCCCCCCCO)NC(CO)CO</smiles>

2-HMMnA<smiles>O=C(CCCCCCCCCCCCCCO)NC(CO)(CO)CO</smiles>

3-HMMnA

Figure 1. Molecular structures of m-HMMnA gelators $(m=1,2,3 ; n=10,12,16)$.

\section{Experimental Section}

Details of the synthetic procedures, purification methods, and characterizations of the m-NMMnA are included in the Supporting Information file. Figures and Tables in that file are prefixed with "S".

Gelation procedures. Preliminary screening for gelation was performed by placing weighed amounts of a gelator and a liquid into a glass tube $(5 \mathrm{~mm}$, i.d.) which was flame-sealed. The mixture was heated to $100{ }^{\circ} \mathrm{C}$ in an oil bath until the solid 
dissolved and a clear solution was obtained. Ice-cooled gels were prepared by placing the hot solutions/sols into an ice-bath for $30 \mathrm{~min}$, then allowing them to stand at room temperature for $2 \mathrm{~h}$. Fast-cooled gels were prepared by removing the hot solutions/sols from the oil bath and leaving them at room temperature for $2 \mathrm{~h}$. Slow-cooled gels were prepared by leaving the hot solutions/sols in the oil bath while it cooled to room temperature after removal of the heating element. In each case, preliminary assignment of the phase formed was based on whether the sample appeared visibly to be mono- or bi-phasic and whether it flowed perceptibly at room temperature when the tube was inverted.

Gel-to-sol transition temperatures $\left(T_{g e l}\right)$ were determined by the "falling ball" method. ${ }^{46}$ A $130 \mathrm{mg}$ steel ball was placed gently at the top of the gel and then the tube containing the gel was flame-sealed. The tube was submerged in a thermostatted water bath starting at room temperature and the temperature was raised slowly $(0.5$ ${ }^{\circ} \mathrm{C} / \mathrm{min}$ ). The temperature range over which gel began to move and then fall to the bottom of the tube was recorded as the $T_{\text {gel }}$ range. Critical gelation concentrations $(C G C s)$ were determined at room temperature $\left(\sim 24{ }^{\circ} \mathrm{C}\right)$ by the "falling drop" method $^{47}$ using a series of fast-cooled gels with $0.1 \mathrm{wt} \%$ concentration increments of LMOG. The minimum concentration of LMOG which formed a stable gel is reported as the $C G C$.

Instrumentation. ${ }^{1} \mathrm{H}$ NMR spectra were acquired on a Varian-MR (400 MHz) spectrometer in $\mathrm{CDCl}_{3}$ or DMSO- $d_{6}$ using tetramethylsilane (TMS) as internal standard. The following abbreviations were used to indicate the peak multiplicities: $\mathrm{s}$ (singlet), d (doublet), $\mathrm{t}$ (triplet) and q (quartet). Elemental analyses were performed on a PerkinElmer $2400 \mathrm{CHN}$ elemental analyzer using acetanilide as a calibration standard. The average of three determinations is reported. Melting temperatures of the neat gelators and polarizing optical micrographs (POMs) of their gels were recorded using a Leitz 585 SM-LUX-POL optical microscope equipped with crossed polars, a Leitz 350 heating stage, a Photometrics CCD camera interfaced to a computer, and an Omega HH503 microprocessor thermometer connected to a J-K-T thermocouple. The 
gel samples for POMs were flame-sealed in $0.5 \mathrm{~mm}$ path-length, flattened Pyrex capillary tubes (VitroCom), heated to their solution/sol phases, and then cooled by one of the cooling processes described above.

Differential scanning calorimetry (DSC) was performed on a TA DSC200 calorimeter in hermetically sealed aluminum pans. Transition temperatures are reported at the onsets of heating endotherms. IR spectra of neat solids were recorded on a Perkin-Elmer Spectrum One FT-IR spectrometer with an attenuated total reflection (ATR) bar. Each spectrum is an average of 32 individual scans at a spectral resolution of $2 \mathrm{~cm}^{-1}$. IR spectra of gels were recorded on a Nicolet 380 FT-IR spectrometer. Hot solutions/sols were injected into a liquid sample cell with $\mathrm{KBr}$ windows and a $0.25 \mathrm{~mm}$ spacer and sealed with Teflon plugs; the samples were allowed to cool to room temperature and remain there for several minutes before spectra were recorded. Powder X-ray diffraction (XRD) of samples sealed in $1.0 \mathrm{~mm}$ glass capillaries (W. Muller, Schonwalde, Germany) were performed on a Rigaku R-AXIS image plate system with $\mathrm{Cu} K_{\alpha}$ X-rays $(\lambda=1.54 \AA$ ) generated by a Rigaku generator operating at $46 \mathrm{kV}$ and $40 \mathrm{~mA}$ with the collimator at $0.5 \mathrm{~mm}$. Materials Data JADE (version 5.0.35) software was used to analyze the data. Diffraction data were collected for $10 \mathrm{~h}$ for gel and solvent samples, and for $2 \mathrm{~h}$ for neat powders.

The viscoelastic properties were studied on a strain-controlled Anton Paar-Physica MCR 301 rheometer equipped with Peltier temperature-controlled stainless steel parallel plates of $25 \mathrm{~mm}$ diameter. Initially, a hot solution/sol was poured onto the lower plate, and the upper plate was lowered, then the sample was sandwiched between the two plates with a gap of $0.6 \mathrm{~mm}$. The plates were heated to $100{ }^{\circ} \mathrm{C}$ and kept at that temperature for 2 min before cooling to $25^{\circ} \mathrm{C}$ for at least 40 min to reform the gel. A solvent trapping device was used to minimize evaporation of the liquids during measurements. Visually, all of the samples remained gels after the measurements had been completed. Data were collected using Rheoplus/32 Service V3.10 software. 
The Hansen solubility parameters (HSPs) of the liquids were available from the literature. ${ }^{48,49}$ Their overall energy density $(\delta)$ was separated into three components: dispersive interactions $\left(\delta_{d}\right)$, polar interactions $\left(\delta_{p}\right)$ and hydrogen bonding interactions $\left(\delta_{h}\right)$ (eq1). ${ }^{43-45}$ Appearances of gelators in different liquids were categorized as soluble $(\mathrm{S})$, gel $(\mathrm{G})$, insoluble $(\mathrm{I})$ and suspension or precipitate $(\mathrm{P})$. All gels were stable for > 2 months when left at room temperature in closed containers.

$\delta=\sqrt{\delta_{p}^{2}+\delta_{h}^{2}+\delta_{d}^{2}}$

\section{RESULTS AND DISCUSSION}

Gelation studies. Unless stated otherwise, experiments were performed on gels made by the fast-cooling method. The gelation abilities of the m-HMMnA at $2 \mathrm{wt} \%$ concentrations were examined in 20 different liquids (Table 1). 1-HMM10A was able to gelate several liquids, such as chlorobenzene and nitrobenzene, but not $n$-alkanes. The m-HMM16A, with the longest alkyl chains examined, showed the best gelation abilities. Thus, 1-HMM16A was able to gelate 11 of the liquids tested, including more polar liquids (such as ethanol) and less polar ones (such as decane). Introducing longer terminal alkyl chains can increase van der Waals attractive forces between gelator molecules as well as their lipophilicity. ${ }^{50,51}$ At the same alkyl chain length, the LMOGs with two hydroxymethyl groups (the 2-HMMnA) were able to gelate more liquids than the 1- or 3-HMMnA. The most efficient gelator, 2-HMM16A, gelated 14 liquids, including $n$-alkanes, aromatic liquids, chloroform, acetonitrile, ethyl acetate, acetone, $\mathrm{CCl}_{4}$ and silicone oil. At very low concentrations $(<1 \mathrm{wt} \%)$, all of the m-HMMnA were able to gelate silicone oil; they are 'supergelators'. In addition, it was possible to gelate layers of silicone oil over water.

All of the m-HMMnA were insoluble in water, even above their melting temperatures, but could be dissolved to different degrees in mixtures of alcohols and DMSO. Thus, within empirically determined volume ratios of DMSO and an alcohol, it was possible to form opaque gels of the m-HMMnA, although no gel could be 
formed in either of the neat liquids (Table S1 and S2). When the bulk liquid properties have the appropriate balance between dissolution and precipitation, the aggregation of the LMOGs within their sol phases can allow a gel network to form. ${ }^{52}$

Table 1. Appearances, ${ }^{\mathrm{a}} T_{\text {gel }}$ values (in parentheses, ${ }^{\circ} \mathrm{C}$ ) of $2 \mathrm{wt} \% \mathrm{~m}-\mathrm{HMMnA}$ in different liquids, and $C G C$ values $(w t \%, \pm 0.1)$ of the gels.

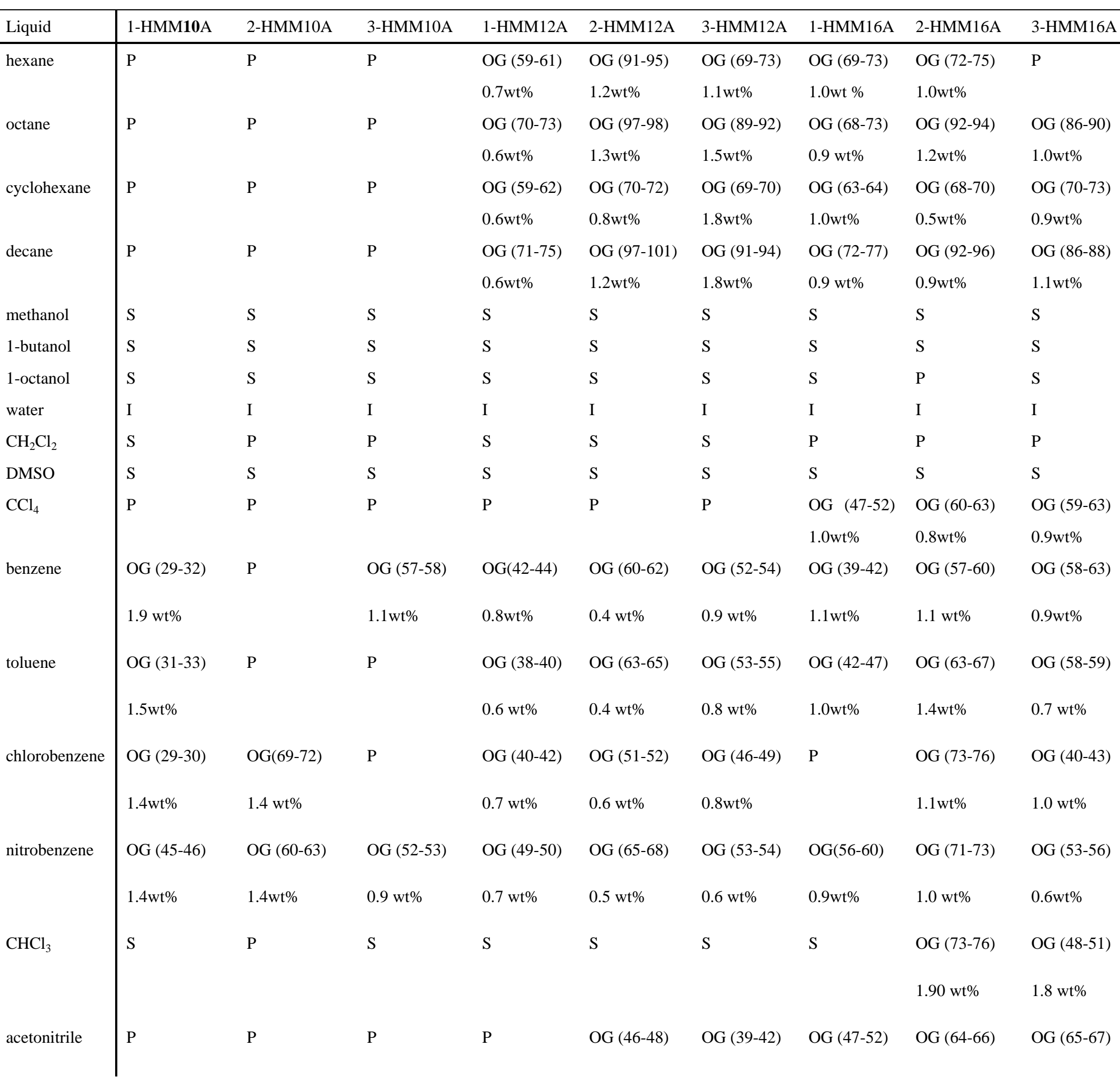




\begin{tabular}{|c|c|c|c|c|c|c|c|c|c|}
\hline & & & & & $0.6 \mathrm{wt} \%$ & $1.0 \mathrm{wt} \%$ & $1.9 \mathrm{wt} \%$ & $1.1 \mathrm{wt} \%$ & $1.1 \mathrm{wt} \%$ \\
\hline \multirow[t]{2}{*}{ ethyl acetate } & $\mathrm{S}$ & $\mathrm{P}$ & $\mathrm{P}$ & $\mathrm{P}$ & OG (35-37) & OG (28-30) & OG (36-38) & OG (60-63) & $\mathrm{P}$ \\
\hline & & & & & $0.8 \mathrm{wt} \%$ & $0.9 \mathrm{wt} \%$ & $1.2 \mathrm{wt} \%$ & $0.6 \mathrm{wt} \%$ & \\
\hline \multirow[t]{2}{*}{ acetone } & $\mathrm{S}$ & $\mathrm{S}$ & $\mathrm{S}$ & $\mathrm{P}$ & OG (42-43) & $\mathrm{P}$ & $\mathrm{P}$ & OG (51-54) & $\mathrm{P}$ \\
\hline & & & & & $0.6 \mathrm{wt} \%$ & & & $1.4 \mathrm{wt} \%$ & \\
\hline \multirow[t]{2}{*}{ silicone oil } & OG (53-55) & OG (85-87) & OG (54-57) & OG (63-66) & OG (85-87) & OG (78-82) & OG (75-76) & OG (97-98) & OG (81-83) \\
\hline & $0.6 \mathrm{wt} \%$ & $0.5 \mathrm{wt} \%$ & $0.5 \mathrm{wt} \%$ & $0.2 \mathrm{wt} \%$ & $0.2 \mathrm{wt} \%$ & $0.4 \mathrm{wt} \%$ & $0.3 \mathrm{wt} \%$ & $0.2 \mathrm{wt} \%$ & $0.3 \mathrm{wt} \%$ \\
\hline
\end{tabular}

${ }^{\mathrm{a}} \mathrm{OG}$ - opaque gel, S - solution, $\mathrm{P}$ - precipitate, I - insoluble.

Gel-sol transition temperatures. As mentioned, all of the gels were stable at room temperature in closed containers for more than two months. To evaluate the thermal stabilities of the gels, gel-sol transition temperatures $\left(T_{g e l}\right)$ of the $2 \mathrm{wt} \%$ m-HMMnA gels were also compared (Table 1). As expected, they are very dependent on the molecular structure of the gelator and the liquid employed. Thus, $T_{g e l}$ values as much as $50{ }^{\circ} \mathrm{C}$ higher were found for gels with $n$-alkanes than with aromatic liquids when the gelators were comprised of dodecyl or hexadecyl chains. Also, $T_{\text {gel }}$ increased with increasing alkyl chain length of the LMOGs, presumably as a result of greater London dispersion forces among neighboring molecules.

Transition temperature ranges of neat $\mathrm{m}-\mathrm{HMMnA}$ and their $5 \mathrm{wt} \%$ nitrobenzene fast-cooled gels were also studied by POM and DSC. DSC-derived melting temperatures (Figure S10) were very reproducible, and they were dependent on both the alkyl length and the number of hydroxymethyl groups (Figure 2). As with the neat powders, the melting temperatures of the nitrobenzene gels increased with $\mathrm{n}$ when $\mathrm{m}$ (i.e., the number of hydroxymethyl groups) was constant in one series of the m-HMMnA. However, at constant values of n (i.e., alkyl chain length), the highest temperatures were observed when $\mathrm{m}$ was 2 . These trends demonstrate the importance of the balance between inter- and intra-molecular H-bonding interactions within the crystalline gel and neat powder networks. 

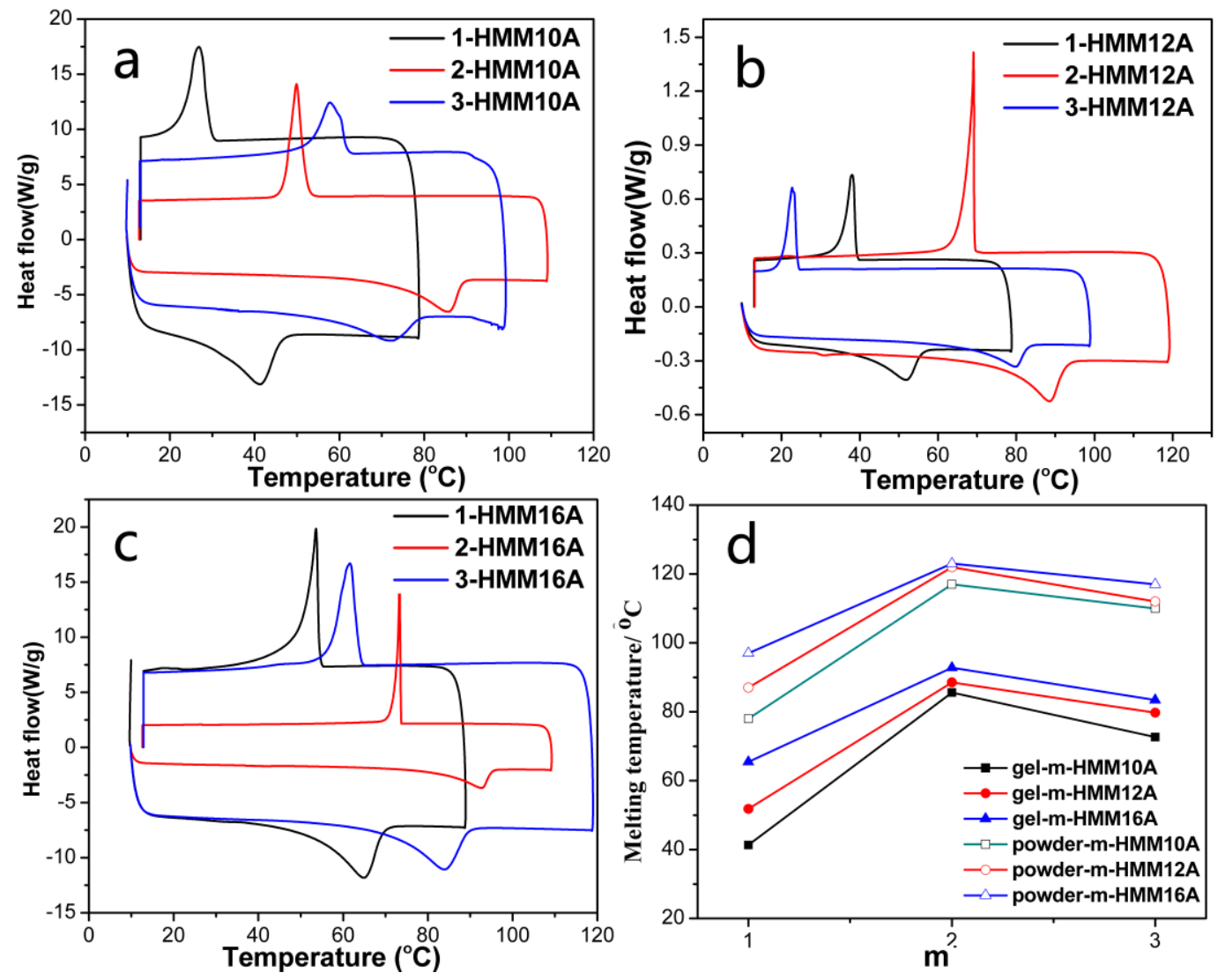

Figure 2. DSC thermograms of 5 wt $\%$ gels formed in nitrobenzene $(a, b, c)$ and comparisons between the melting temperatures of the neat gelators and their gels (d); $\mathrm{m}$ is the number of hydroxymethyl groups attached to the carbon atom adjacent to nitrogen.

The interactions between gelator molecules must be balanced against gelator-liquid interactions within the gels (as interfacial energies) and in the sol phases as the gels are forming (as solvent-solute energies). One convenient method to explore these interactions is Hansen solubility parameters which separate solvent properties into dispersive interactions $\left(\delta_{d}\right)$, polar interactions $\left(\delta_{p}\right)$ and hydrogen bonding interactions $\left(\delta_{h}\right)$ (Figures 3, 4 and S11-14). ${ }^{53,54}$ However, although Hansen parameters (and other interaction parameters related to them) are a very valuable diagnostic of gelator-liquid interactions, they do not separate the magnitudes and relative importance of those interactions at the various stages of transformation of a sol to a gel phase. For that reason, one should not expect Hansen (or other treatments) to yield useful correlations in those cases where the interactions at different stages 
leading to gels are physically distinct. Thus, no obvious correlation was observed between $T_{g e l}$ values and $\delta_{d}, \delta_{p}$, and the overall $\delta$. However, some correlation between $T_{g e l}$ values and $\delta_{h}$ was apparent $\left(\mathrm{R}^{2},=0.927\right)$. Generally, $T_{g e l}$ values decreased with increasing $\delta_{h}$, indicating again the importance of $\mathrm{H}$-bonding interactions between the liquid and the gelator at the critical stage(s) of SAFIN formation. A similar trend was found for 3-HMM12A (Figure 4). However, the CGC values with 3-HMM12A showed only a very rough correlation with $\delta_{d}\left(\mathrm{R}^{2}=0.636\right)$, indicative of the limited importance of interfacial interactions.
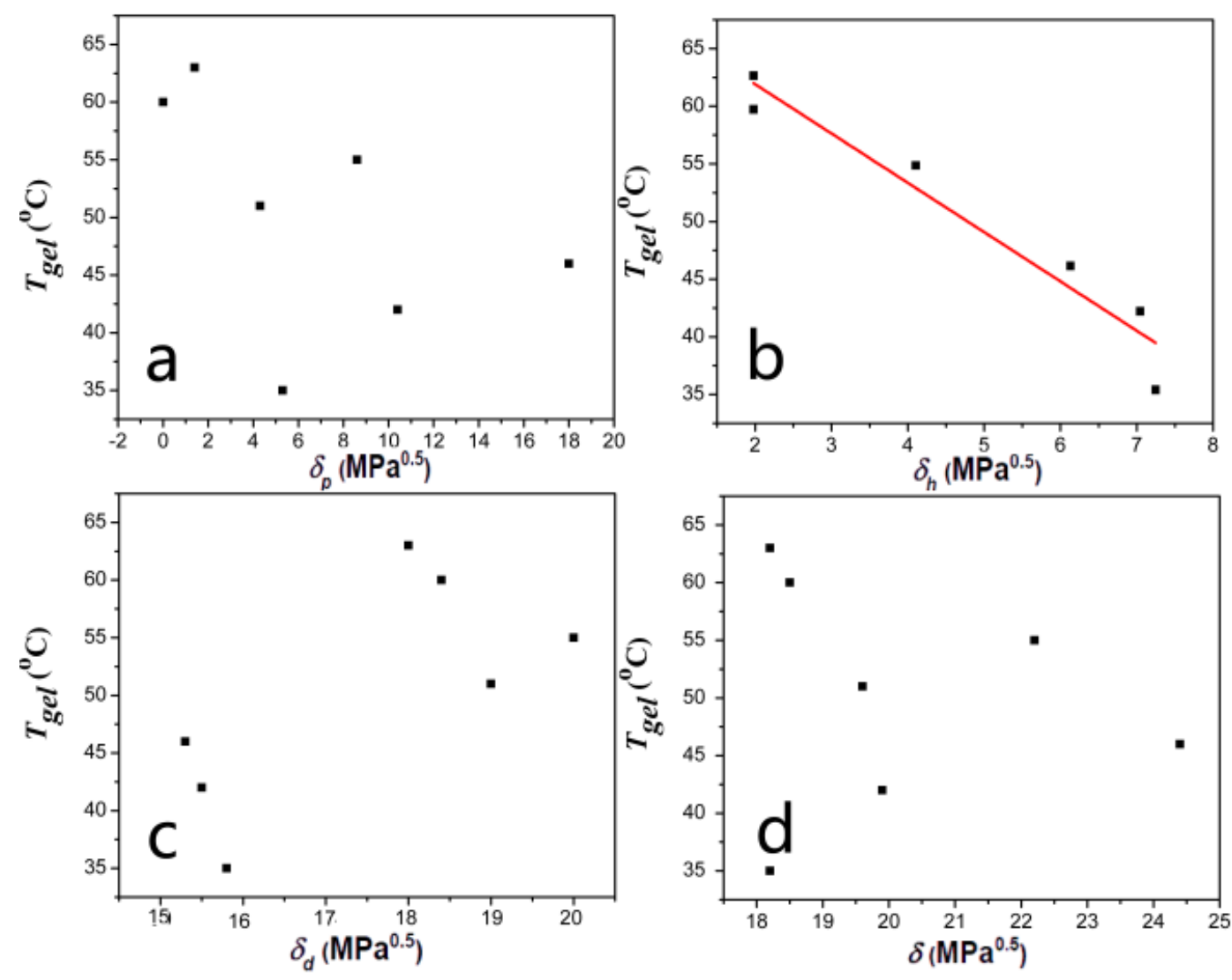

Figure 3. Gel-sol transition temperatures of $2 \mathrm{wt} \%$ 2-HMM12A gels after their preparation by the fast-cooling process as a function of (a) $\delta_{p}$, (b) $\delta_{h}$, (c) $\delta_{d}$, and (d) overall $\delta$ in Hansen space. 

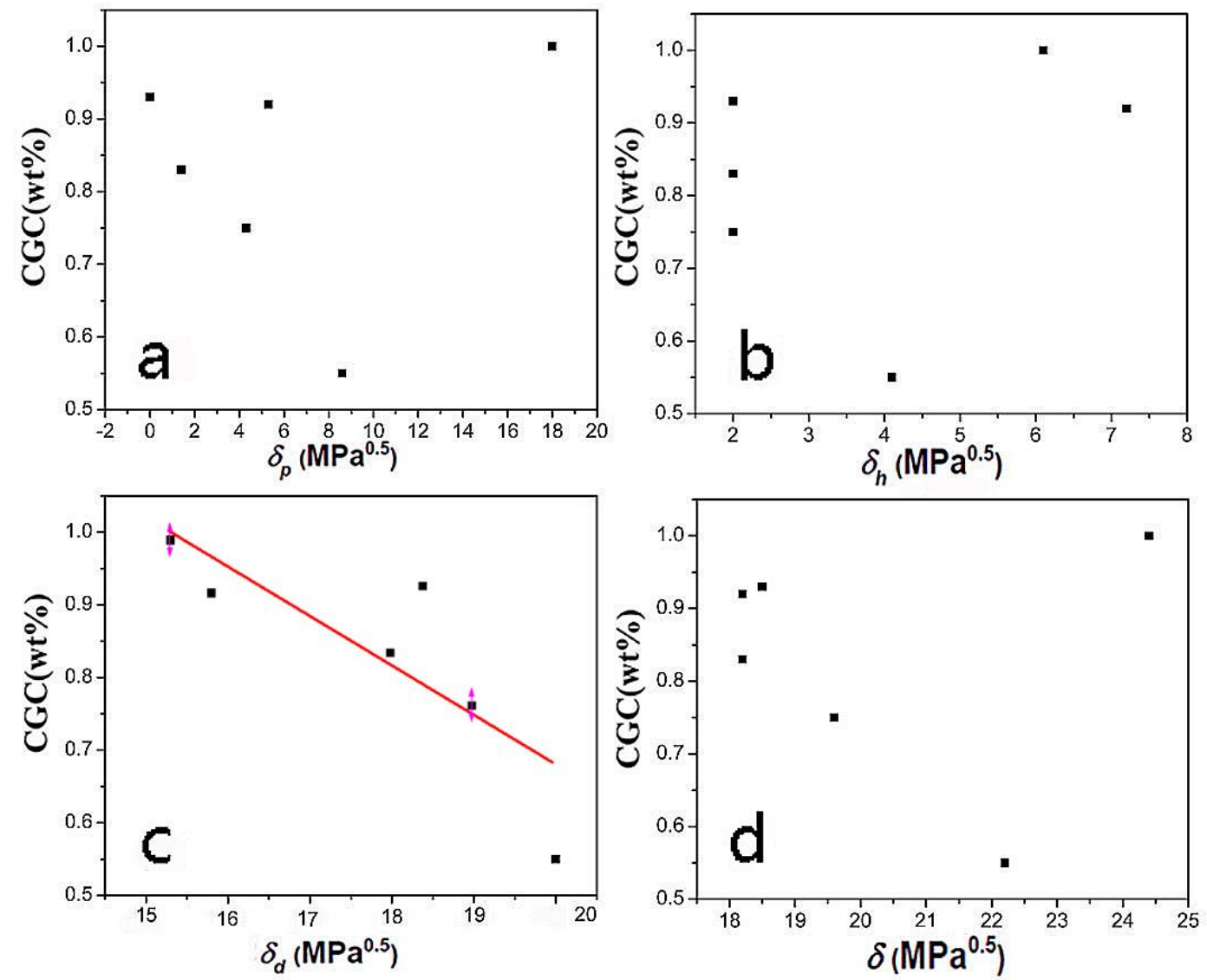

Figure 4. Critical gelation concentrations ( $C G C \mathrm{~s}$ ) of 3-HMM12A gels as a function of (a) $\delta_{p}$, (b) $\delta_{h}$, (c) $\delta_{d}$, and (d) overall $\delta$ in Hansen space.

Morphologies from POM measurements. The self-assembled structures of the gels, prepared from the sol phases using the three different cooling methods, were examined by POM (Figures 5-7) and the sizes of the constituent objects are summarized in Table S3. In many cases, the objects shown must be composed of much smaller units that cannot be discerned at the magnifications employed. Regardless, the data presented demonstrate the wide diversity of SAFIN structures that can be made by modifying the protocol for converting a sol of one of the m-HMMnA into its gel phase.

The gels of $2 \mathrm{wt} \%$ 1-HMM10A in nitrobenzene (Figure 5a-c) showed well-defined 3D networks consisting of many entangled, long fibers with $\sim 9-11 \mu \mathrm{m}$ diameters. Different cooling procedures had little apparent effect on these assembled structures at the magnifications employed. Similar conclusions were also drawn from the POM images of the gels from 2-HMM16A and 3-HMM16A in nitrobenzene and silicone oil 
(Figures S17 and S18).

However, the importance of the cooling protocol used to convert the sols to gels on the shapes of the eventual objects formed is evident in other gel systems. For example, the images of the SAFINs of 1-HMM10A in silicone oil (Figure 5d-f), 3-HMM12A in toluene (Figure 5g-i) and 1-HMM16A in silicone oil (Figure S16) are drastically different depending on the cooling protocol. When prepared by the slow-cooling protocol, the objects from 2-HMM16A in toluene consisted of plate-like structures (Figure 6f) whose lengths and widths exceeded $100 \mu \mathrm{m}$, but were long fibers when the gels were prepared from their sols by the ice-cooling $(\sim 2-5 \mu \mathrm{m}$ diameters) and fast-cooling methods ( 5-10 $\mu$ m diameters) (Figurs 6d-e). SAFINs of the toluene gels of 3-HMM12A (Figure 5g-i)and 3-HMM16A (Figure 6g-i), prepared by the ice-cooling protocol, consisted of spherulites (Figure $5 \mathrm{~g}$ and $6 \mathrm{~g}$ ) whose diameters were about $1 \mathrm{~mm}$ and $<100 \mu \mathrm{m}$, respectively. Those prepared by the ice-cooling and fast-cooling protocols showed only fibers with $\sim 8-10 \mu \mathrm{m}$ and $\sim 3-4$ $\mu \mathrm{m}$ diameters, respectively. Similar dependencies of SAFIN structures on the mode of gel preparation from sol phases have been observed in other systems. ${ }^{46,55-57}$

The 2-HMM16A in nitrobenzene gel prepared, by the fast-cooling protocol showed helical fibers (Figure 6a,b). Despite the molecular chirality of the gelator, both clockwise and counter-clockwise twisted fibers, with $\sim 5 \mu \mathrm{m}$ diameters and non-uniform pitches ranging from 25 to $36 \mu \mathrm{m}$, were present. It is known that helical structures are possible even when the gelator or liquid is not chiral. ${ }^{58}$ Furthermore, as exemplified by the SAFINs of 3-HMM12A in nitrobenzene (Figure 7), the objects in the SAFINs can be manipulated by combining two different cooling methods. 


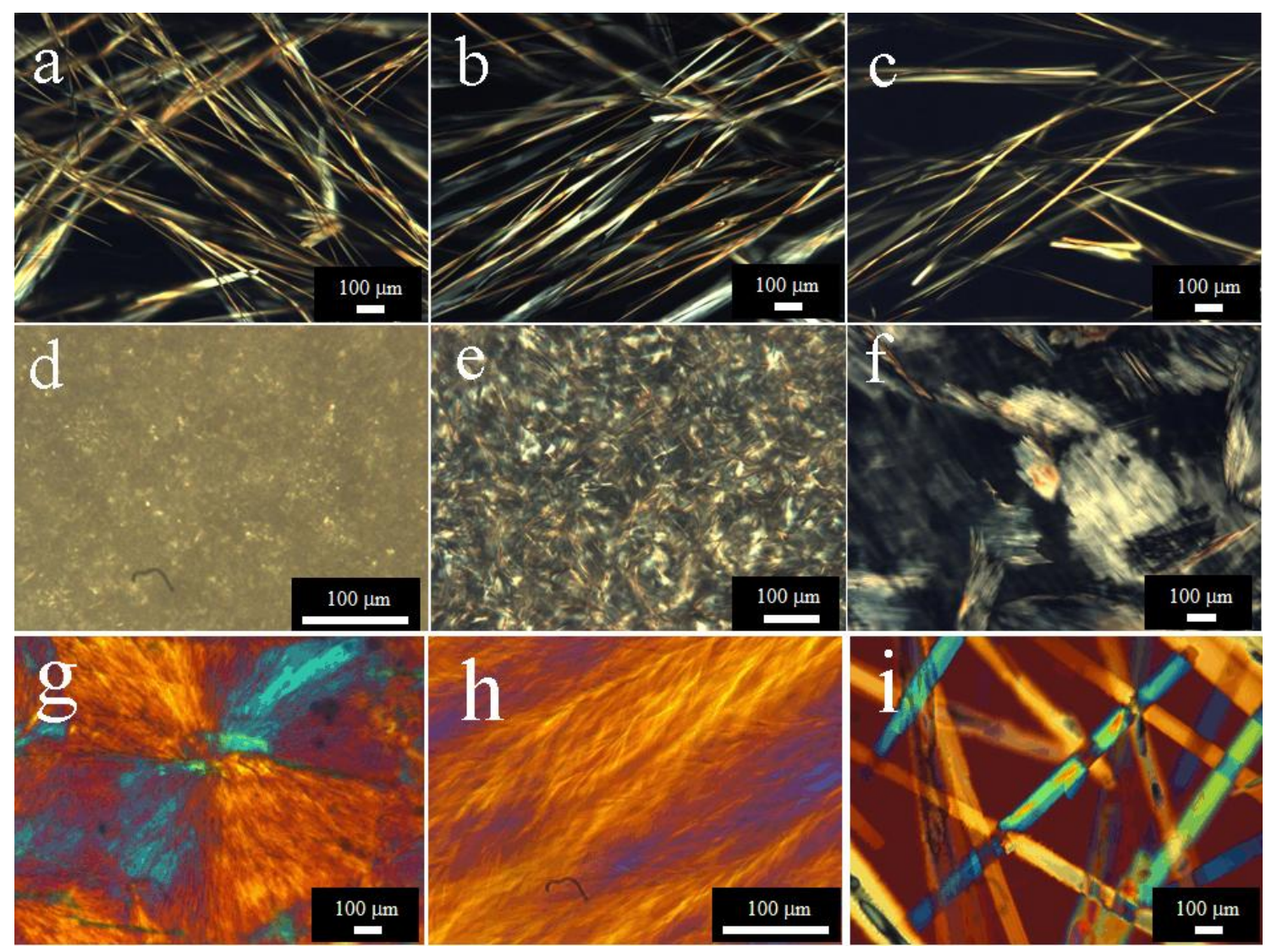

Figure 5. Polarizing optical micrographs at $23{ }^{\circ} \mathrm{C}$ of gels of $2 \mathrm{wt} \% 1-\mathrm{HMM} 10 \mathrm{~A}$ in nitrobenzene (a-c), 2 wt\% 1-HMM10A in silicone oil (d-f), and $2 \mathrm{wt} \%$ 3-HMM12A in toluene (g-i) prepared by the ice-cooling (a, d, g), fast-cooling (b, e, h) and slow-cooling (c, f, i) protocols. 


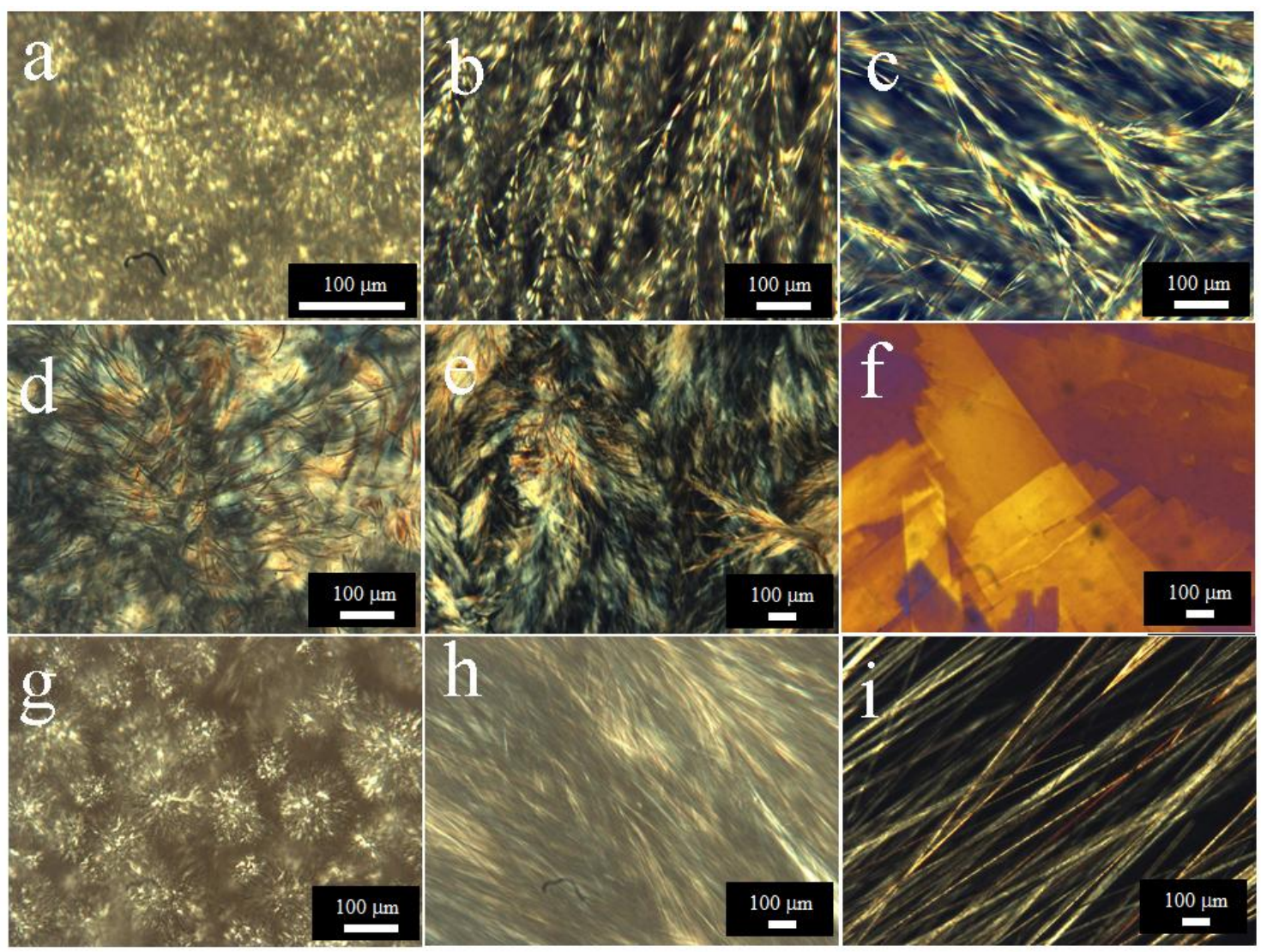

Figure 6. Polarizing optical micrographs at $23{ }^{\circ} \mathrm{C}$ of gels of $2 \mathrm{wt} \%$ 2-HMM16A in nitrobenzene (a-c), 2 wt\% 2-HMM16A in toluene (d-f), and $2 \mathrm{wt} \%$ 3-HMM16A in toluene (g-i) prepared by the ice-cooling (a, d, g), fast-cooling (b, e, h) and slow-cooling (c, f, i) protocols.
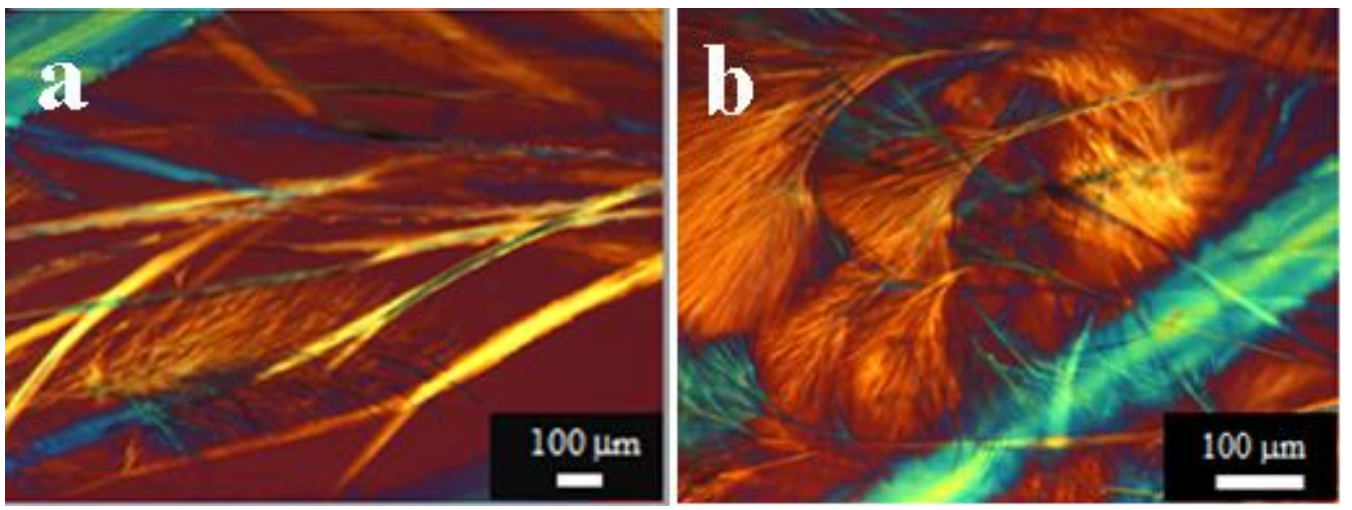

Figure 7. Polarizing optical micrographs at $23{ }^{\circ} \mathrm{C}$ of gels of $2 \mathrm{wt} \% 3-\mathrm{HMM} 12 \mathrm{~A}$ in nitrobenzene that were slow-cooled to $40{ }^{\circ} \mathrm{C}$ and then fast-cooled to $23{ }^{\circ} \mathrm{C}$ (a) or slow-cooled to $50{ }^{\circ} \mathrm{C}$ and then fast cooled to $23{ }^{\circ} \mathrm{C}(\mathrm{b})$.

As found here, the branching patterns of the self-assembled structures of a gel are 
influenced significantly by changes in the incubation temperature or rate of cooling of the hot sols (i.e., the thermodynamic driving force for SAFIN formation when sols are cooled to $\left.<T_{g e l}\right) .{ }^{17,18,59-63}$ Generally, higher cooling rates or lower incubation temperatures favor formation of more highly branched fibrillar networks or spherulites; lower cooling rates and higher incubation temperatures lead to gel networks with less branched objects because the growth of nucleating OD and 1D objects is more discriminate. ${ }^{64}$

Mechanical properties. The viscoelastic properties of the gels in nitrobenzene were studied quantitatively by rheology (Figures 8 and S19). The storage modulus $\left(G^{\prime}\right)$ remained higher than the loss modulus $\left(G^{\prime \prime}\right)^{65}$ within the linear viscoelastic region (LVR), and both moduli were independent of the frequency over the range examined at $0.1 \%$ strain (i.e., within the LVR). These are the characteristics of true gels. ${ }^{3,4,66}$ Both $G^{\prime}$ and $G^{\prime \prime}$ values increased with increasing gelator concentration (as expected for stronger and more extensive SAFINs). The critical strain values (i.e., where the values of $G^{\prime}$ and $G^{\prime \prime}$ cross and above which the materials are no longer gels) are collected in Table 2. Thus, the gels of $5 \mathrm{wt} \%$ 1-HMM12A, 2-HMM12A, and 3-HMM12A in nitrobenzene exhibited crossover strains of $0.18,1.59$, and $0.87 \%$, respectively: the 1-HMM12A gel was the weakest mechanically, and the gel of $5 \mathrm{wt} \%$ 3-HMM12A in nitrobenzene had the largest $G^{\prime}$ value $(0.34 \mathrm{MPa})$ at $0.01 \%$ strain and $1 \mathrm{~Hz}$ frequency. Generally (but with some exceptions), the mechanical strengths of the $5 \mathrm{wt} \% \mathrm{~m}-\mathrm{HMMnA}$ in nitrobenzene gels increased as the number of hydroxymethyl groups became larger or the alkyl chain length became longer. An exception to this generalization is found in the $5 \mathrm{wt} \% \mathrm{~m}-\mathrm{HMM} 16 \mathrm{~A}$ in nitrobenzene gels where the value of $G^{\prime}$ for 1-HMM16A, 1.07 MPa, was much larger than that for 3-HMM16A, $0.25 \mathrm{MPa}$. 

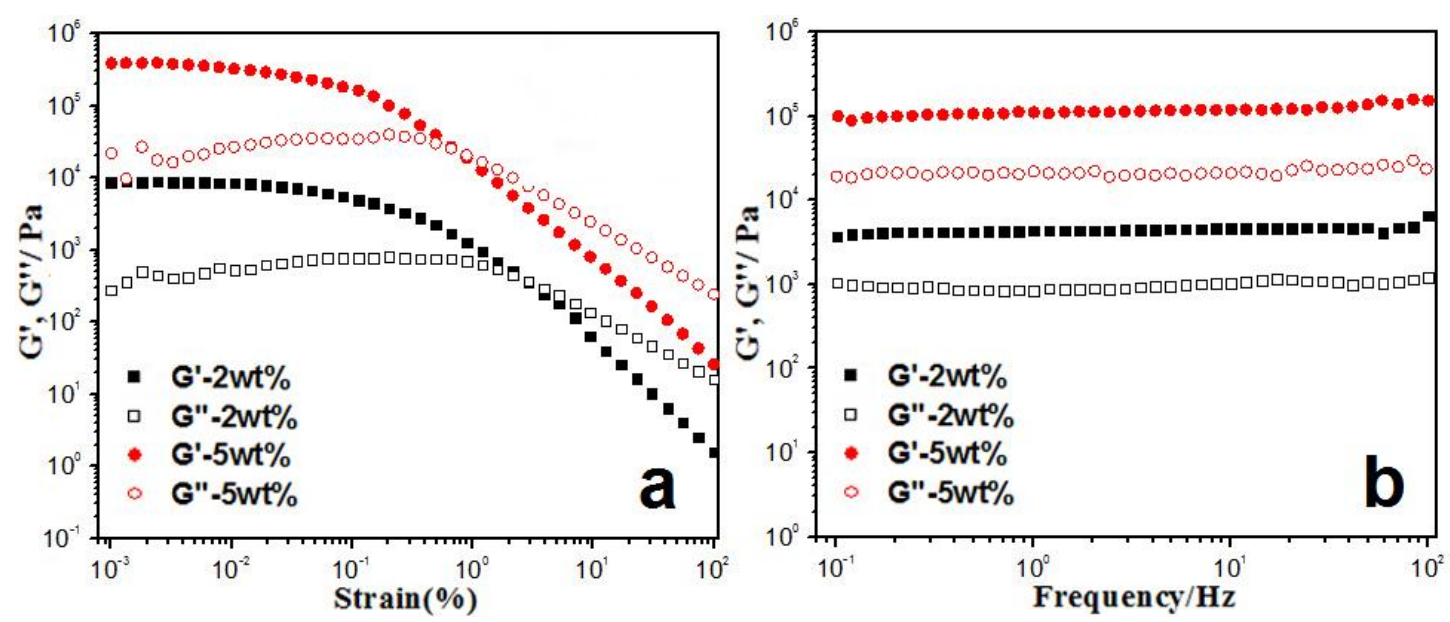

Figure 8. Log-log shear strain sweeps (frequency $=1 \mathrm{~Hz}$ ) (a) and frequency sweeps (strain=0.1\%) (b) for gels of $2(\boldsymbol{\square}, \square)$ and $5 \mathrm{wt} \%(\boldsymbol{O}, \bigcirc)$ 3-HMM12A in nitrobenzene. $G^{\prime}$, closed symbols; $G^{\prime \prime}$, open symbols.

Table 2. $G^{\prime}$ and $G^{\prime \prime}$ values and $G^{\prime} / G^{\prime \prime}$ ratios at strain $=0.1 \%$, frequency=1 $\mathrm{Hz}$, and crossover strain values of $5 \mathrm{wt} \% \mathrm{~m}-\mathrm{HMMnA}$ in nitrobenzene gels at $25^{\circ} \mathrm{C}$.

\begin{tabular}{lcccc}
\hline & $G^{\prime}$ & $G^{\prime \prime}$ & $G^{\prime} / G^{\prime \prime}$ & Crossover \\
& & & & strain (\%) \\
\hline 1-HMM10A & 59400 & 14700 & 4.04 & 16.8 \\
2-HMM10A & 34300 & 2900 & 11.8 & 0.24 \\
3-HMM10A & 237000 & 52800 & 4.49 & 0.35 \\
1-HMM12A & 89500 & 13000 & 6.88 & 0.18 \\
2-HMM12A & 261000 & 26500 & 9.85 & 1.59 \\
3-HMM12A & 340000 & 24200 & 14.0 & 0.87 \\
1-HMM16A & 1070000 & 62400 & 17.1 & 0.29 \\
2-HMM16A & 249000 & 8740 & 28.5 & 0.67 \\
3-HMM16A & 251000 & 14900 & 16.8 & 0.35 \\
\hline
\end{tabular}

Hydrogen-bonding interactions from analyses of FT-IR spectra. FT-IR 
spectra provide valuable information about the nature of the hydrogen-bonding within the neat solid and SAFINs of the gels (Figure S20-S27). Unfortunately, the hydroxyl stretching regions for all of the solids and gels examined, $3150-3400 \mathrm{~cm}^{-1}$, were rather complex although they indicated extensive H-bonding. ${ }^{67}$ As expected, the spectra indicate that $-\mathrm{NH}$ and $-\mathrm{CO}$ groups are involved in $\mathrm{H}$-bonding interactions as well. The similarity between the frequencies of the pertinent bands of the gelator powders and their corresponding gels suggests similar packing arrangements, at least near the head groups of the LMOGs. In addition, a band near $1470 \mathrm{~cm}^{-1}$ was observed in the neat powder and gel phases. It is a characteristic of scissoring $\mathrm{CH}_{2}$ absorptions in which several contiguous methylenes are in an all-trans conformation. ${ }^{68,69}$

\section{Molecular packing arrangements from powder X-ray diffraction data.} Powder XRD was employed to gain further insights into the packing arrangements of the $\mathrm{n}$-HMM12A gelators in their fast-cooled $5 \mathrm{wt} \%$ gels in nitrobenzene and the neat powders recrystallized from acetone (Figures S28-S30), including whether the arrangements differ in the two phases. The corresponding $d$ values for the diffraction peaks were calculated using the Bragg equation (Table S3). Only for 3-HMM12A is the distance corresponding to the lowest angle peak, $22.9 \AA$, similar to the extended molecular length of a molecule, $\sim 24 \AA$, as calculated by the MM2 method. Sufficient numbers of diffractions were found to index the low angle peaks and to make provisional assignments of cell packings for the neat m-HMM12A: ${ }^{26} 1$-HMM12A (Figure S28) can be indexed to a monoclinic cell ( $\mathrm{a}=40, \mathrm{~b}=5, \mathrm{c}=9 \AA$ ) ; 2-HMM12A (Figure S29) fits a monoclinic cell as well ( $a=8, b=6, c=32 \AA)$; and the cell of 3-HMM12A is triclinic $(a=10, b=10, c=23 \AA)$. Although the diffractions of the m-HMM12A in their gel phases were not as well defined, their patterns were very similar to those of the neat powders. On that basis, we ascribe tentatively the packing arrangements in the two phases to be the same..$^{26,70}$

Despite that correspondence, there was no clear relationship between the melting temperatures of the neat gelators (see Supporting Information) and those of the $2 \mathrm{wt} \%$ m-HMM12A gels in nitrobenzene (Table 1), despite the fact that the $C G C$ values for 
the 3 gels, 0.5-0.7 wt\%, are very similar (i.e., the amount of gelator within the SAFINs is nearly the same at room temperature). We ascribe this observation to the differences discussed above among the sizes and shapes of the elements constituting the SAFIN structures; different gel properties depend on structural factors at different distance scales.

Thixotropic properties. Qualitatively, the gels of all of the m-HMMnA were thixotropic in nitrobenzene (Figures S31-S127) and toluene. Because toluene is quite volatile, quantitative rheological information was recorded only for the nitrobenzene gels.

The data in Figure S31 indicate that viscoelastic recovery of 1-HMM10A in nitrobenzene is much slower than for the other gels examined. Consistent with those data, a bulk sample did not reform a gel phase even several minutes after being broken by vigorous shaking. Thus, the driving force for reassembly of the pieces of this SAFIN after application of destructive strain is much lower than for many of the other m-HMMnA. In a more detailed examination, a 2 wt\% 1-HMM12A in nitrobenzene gel was placed under constant frequency $(1 \mathrm{~Hz})$ and strain $(0.10 \%)$ for $420 \mathrm{~s}$ to give initial values of $G^{\prime}(3047 \mathrm{~Pa})$ and $G^{\prime \prime}(866 \mathrm{~Pa})$ (Figure 9). The isothermal gel-to-sol transition was achieved by shearing the sample at $80 \%$ strain for $180 \mathrm{~s}$. Under these conditions, $G$ ' was reduced to ca. $0.1 \mathrm{~Pa}$, a value smaller than $G$ ”' (ca. 1 $\mathrm{Pa}$ ); the gel had been broken. Then, the degree and time of gel recovery were followed by measuring the temporal evolution of the moduli commencing immediately after changing the strain again to $0.10 \%$. About $96 \%$ of the original $G^{\prime}$ value was recovered within $20 \mathrm{~s}$. At least $82 \%$ of the original $G^{\prime}$ value was recovered after 4 additional high strain-low strain cycles. 


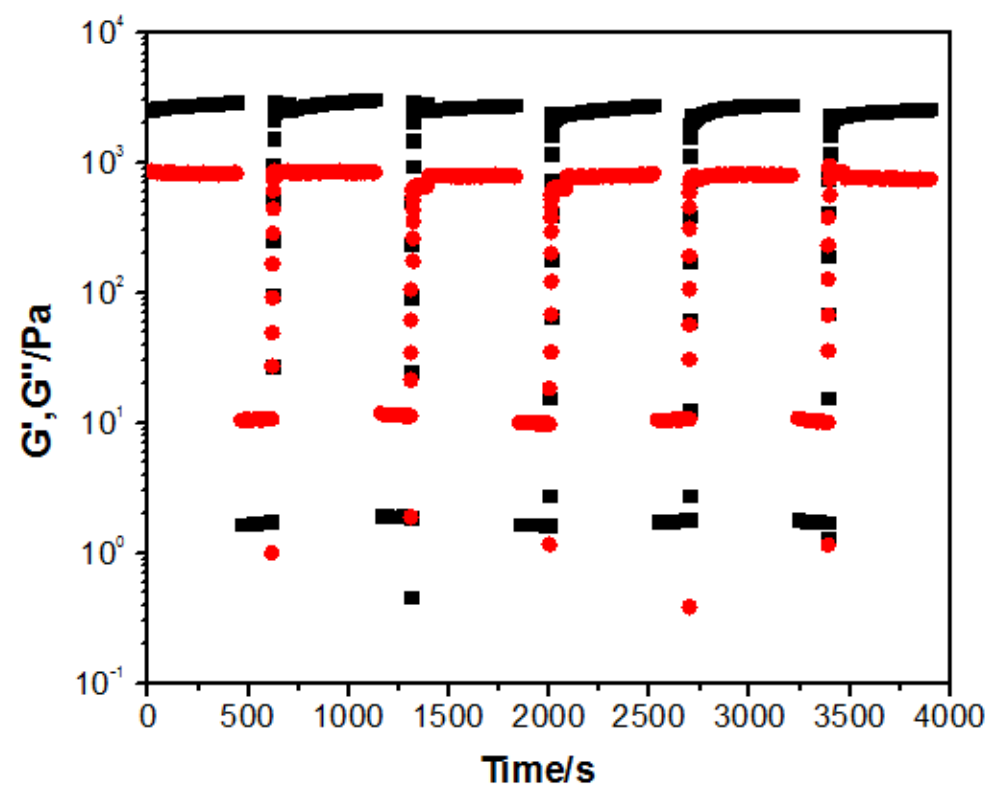

Figure 9. $G^{\prime}$ (black) and $G^{\prime \prime}$ (red) of a gel of $2 \mathrm{wt} \%$ 1-HMM12A in nitrobenzene as a function of time at different strains and a frequency of $1 \mathrm{~Hz}$. The initial strain, $0.10 \%$, and destructive strain, $80 \%$, were changed sequentially as indicated.

The effects of molecular structure, gelator concentration, and percent of destructive strain applied on the recovery of several gels were also examined (Table 3). In general, the recovery rates were very fast and the amount of destructive strain had little effect on the degree of recovery. Although the concentration of m-HMM10A or m-HMM16A in their nitrobenzene gels had very little effect on their degree of recovery (within the concentration range explored), there was a marked dependence on the concentration of the m-HMM12A that was acutely sensitive to the number of hydroxymethyl groups: increasing the concentration from 2 to $5 \mathrm{wt} \%$ of 1-HMM12A in nitrobenzene decreased the degree of recovery from 80 to $40 \%$. Although a similar dependence was found for 3-HMM12A, the recovery increased from 80 to $90 \%$ as the concentration of 2-HMM12A gel was increased from 2 to $5 \mathrm{wt} \%$. This trend is consistent with the crossover strain changes as gelator concentration is increased (Figure S128). These results indicate that, with the exception of the nitrobenzene gels of 1-HMM10A, weaker SAFINs are able to recover more easily after destructive strain than stronger ones, perhaps as a result of greater intrinsic mobility of the former. 
Table 3. Degrees of recovery of $G^{\prime}$ after 5 cycles at different destructive strains for 2 and $5 \mathrm{wt} \% \mathrm{~m}-\mathrm{HMMnA}$ in nitrobenzene gels.

\begin{tabular}{|c|c|c|c|c|}
\hline \multirow[t]{2}{*}{ m-HMMnA } & \multirow[t]{2}{*}{ concn } & \multicolumn{3}{|c|}{ destructive strain $^{\mathrm{a}}$} \\
\hline & & $20 \%$ & $80 \%$ & $100 \%$ \\
\hline \multirow[t]{2}{*}{$1-\mathrm{HMM} 10 \mathrm{~A}^{\mathrm{b}}$} & $2 \mathrm{wt} \%$ & $\mathrm{X}$ & $\mathrm{X}$ & $\mathrm{X}$ \\
\hline & $5 \mathrm{wt} \%$ & $X$ & $X$ & $X$ \\
\hline \multirow[t]{2}{*}{ 2-HMM10A } & $2 \mathrm{wt} \%$ & 83 & 82 & 79 \\
\hline & $5 \mathrm{wt} \%$ & 83 & 81 & 79 \\
\hline \multirow[t]{2}{*}{ 3-HMM10A } & $2 \mathrm{wt} \%$ & 76 & 81 & 76 \\
\hline & $5 \mathrm{wt} \%$ & 69 & 71 & 73 \\
\hline \multirow[t]{2}{*}{ 1-HMM12A } & $2 \mathrm{wt} \%$ & 76 & 82 & 79 \\
\hline & $5 \mathrm{wt} \%$ & 41 & 42 & 43 \\
\hline \multirow[t]{2}{*}{ 2-HMM12A } & $2 \mathrm{wt} \%$ & 76 & 81 & 76 \\
\hline & $5 \mathrm{wt} \%$ & 86 & 92 & 90 \\
\hline \multirow[t]{2}{*}{ 3-HMM12A } & $2 \mathrm{wt} \%$ & 92 & 93 & 92 \\
\hline & $5 \mathrm{wt} \%$ & 67 & 64 & 71 \\
\hline \multirow[t]{2}{*}{ 1-HMM16A } & $2 \mathrm{wt} \%$ & 90 & 90 & 84 \\
\hline & $5 \mathrm{wt} \%$ & 82 & 84 & 80 \\
\hline \multirow[t]{2}{*}{ 2-HMM16A } & $2 \mathrm{wt} \%$ & 99 & 92 & 92 \\
\hline & $5 \mathrm{wt} \%$ & 93 & 93 & 91 \\
\hline \multirow[t]{2}{*}{ 3-HMM16A } & $2 \mathrm{wt} \%$ & 92 & 93 & 84 \\
\hline & $5 \mathrm{wt} \%$ & 92 & 93 & 93 \\
\hline
\end{tabular}

${ }^{a}$ Values in columns are the degrees of recovery. ${ }^{b} \mathrm{X}$ indicates no or very slow recovery after cessation of destructive strain.

The degree of recovery after each cycle was also plotted (Figures 10 and S129). For example, for the gel of $2 \mathrm{wt} \% 3$-HMM10A in nitrobenzene (Figure 10a), $G^{\prime}$ decreased to $77 \%$ after one cycle (destructive strain $20 \%$ ) and remained virtually the 
same during the subsequent 4 cycles. The gels of 2-HMM10A, 3-HMM10A, 3-HMM12A and 2-HMM16A in nitrobenzene behaved similarly. We hypothesize that there are changes in the surface interactions between the plate of the rheometer and the gel during the first cycle that lead to the decreased $G^{\prime}$ value. The comportment of the other gels is exemplified by the gel of $2 \mathrm{wt} \%$ 2-HMM12A in nitrobenzene (Figure 10 b), where $G^{\prime}$ decreased gradually after each cycle (destructive strain $80 \%$ ) and fell to $81 \%$ of the initial value after 5 cycles.
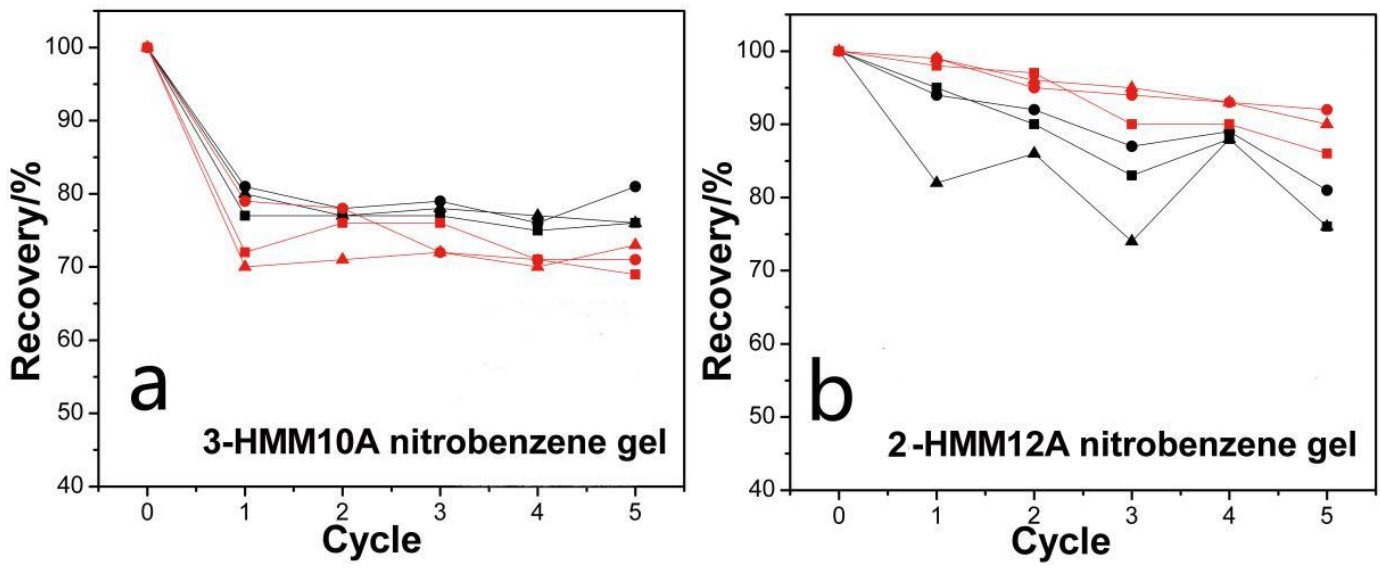

Figure 10. Recovery of $G^{\prime}$ over the first 5 cycles with 2 wt $\%$ (black symbols) and 5 wt $\%$ (red symbols) (a) 3-HMM10A and (b) 2-HMM12A in nitrobenzene gels. Gel samples under $20 \%(\boldsymbol{\square}, \boldsymbol{\square}), 80 \%(\boldsymbol{O}, \mathbf{O})$ and $100 \%$ destructive strain $(\boldsymbol{\Delta}, \boldsymbol{\Delta})$.

Figure 11 shows the temporal evolution of the storage modulus $G^{\prime}$ (average of 5 cycles) after the cessation of destructive strain. The $G^{\prime}$ values were very small initially, indicating the samples to be more viscous than elastic. Thereafter, $G^{\prime}$ increased rapidly, and gradually reached plateau values that were much larger than the $G^{\prime \prime}$ values.

A stretched exponential model (eq 2) was used to analyze further the data. ${ }^{71}$ In it, $\tau$ is the viscoelastic recovery time (which depends on the reformation probability of crosslinks $^{72}$ ) and $m$ is the stretching exponent for the distribution of the relaxation times, $\tau^{73,74}$

$$
\ln \left[-\ln \frac{G^{\prime}(\infty)-G^{\prime}(t)}{G^{\prime}(\infty)-G^{\prime}(0)}\right]=m \ln t-m \ln \tau
$$



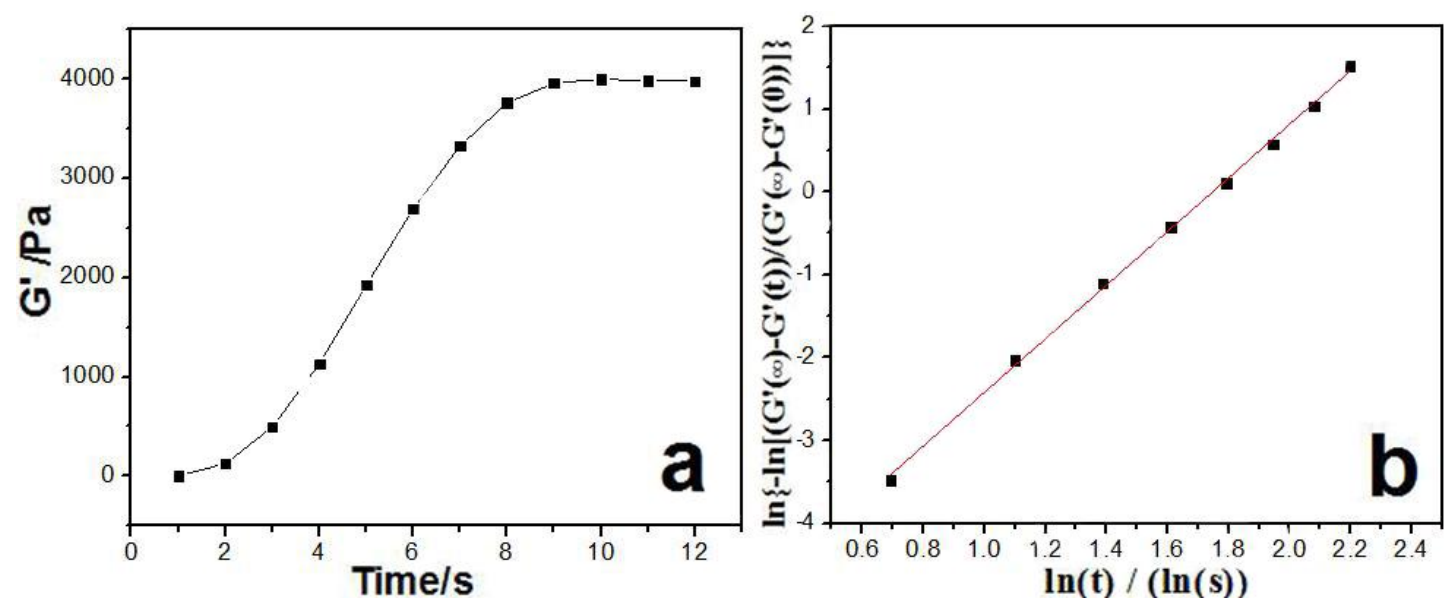

Figure 11. (a) Recovery of $G^{\prime}$ using the averaged data from 5 consecutive recovery cycles and (b) linear fits of the data in (a) to the recovery time of $G^{\prime}\left(\mathrm{R}^{2}=0.999\right)$ $(\mathrm{y}=3.2 \ln t-5.7)$ of a fast-cooled 2 wt $\% 3$-HMM12A in nitrobenzene gel at $25{ }^{\circ} \mathrm{C}$ recorded in the linear viscoelastic regime $(\mathrm{LVR}$, strain $=0.1 \%$, frequency $=1 \mathrm{~Hz})$ after applying a destructive strain of $80 \%$ (frequency $=1 \mathrm{~Hz}$ ). The value of $\tau$ is $7.5 \pm 1.5 \mathrm{~s}$.

For the 2 wt $\%$ 2-HMM10A in nitrobenzene gel (20\% destructive strain), $\tau$ was calculated to be ca. $9 \mathrm{~s}$. The effects of molecular structure, gelator concentration, and percent of destructive strain on the recovery time were studied as well (Table 4). In each case, the recovery time was very short, between 5 and $12 \mathrm{~s}$.

Table 4. Recovery times of $G$ ' after 5 cycles at different destructive strains for 2 and 5 wt\% m-HMMnA in nitrobenzene gels.

\begin{tabular}{ccccc}
\hline molecules & concn & \multicolumn{3}{c}{ destructive strain $^{\mathrm{a}}$} \\
& & $20 \%$ & $80 \%$ & $100 \%$ \\
\hline 1-HMM10A & $2 \mathrm{wt} \%$ & $\mathrm{X}$ & $\mathrm{X}$ & $\mathrm{X}$ \\
& $5 \mathrm{wt} \%$ & $\mathrm{X}$ & $\mathrm{X}$ & $\mathrm{X}$ \\
& & & & \\
& & & & \\
& & & & \\
& $5 \mathrm{wt} \%$ & $7.6 \pm 0.78 \mathrm{~s}$ & $10 \pm 1.2 \mathrm{~s}$ & $11 \pm 1.6 \mathrm{~s}$ \\
3-HMM10A & $2 \mathrm{wt} \%$ & $8.5 \pm 0.34$ & $11 \pm 1.1 \mathrm{~s}$ & $12 \pm 0.1 \mathrm{~s}$ \\
& $5 \mathrm{wt} \%$ & $7.7 \pm 0.54$ & $9.7 \pm 2.1 \mathrm{~s}$ & $10 \pm 3.1 \mathrm{~s}$ \\
& $2 \mathrm{wt} \%$ & $5.9 \pm 0.23 \mathrm{~s}$ & $7.5 \pm 0.45 \mathrm{~s}$ & $7.8 \pm 0.62 \mathrm{~s}$
\end{tabular}




$\begin{array}{ccccc} & 5 \mathrm{wt} \% & 7.2 \pm 1.2 \mathrm{~s} & 8.9 \pm 1.8 & 9.1 \pm 1.5 \mathrm{~s} \\ \text { 2-HMM12A } & 2 \mathrm{wt} \% & 5.8 \pm 0.87 \mathrm{~s} & 7.5 \pm 1.5 \mathrm{~s} & 7.9 \pm 0.71 \mathrm{~s} \\ & 5 \mathrm{wt} \% & 5.8 \pm 0.52 \mathrm{~s} & 7.4 \pm 0.21 \mathrm{~s} & 8.0 \pm 2.5 \mathrm{~s} \\ \text { 3-HMM12A } & 2 \mathrm{wt} \% & 4.9 \pm 0.25 \mathrm{~s} & 5.8 \pm 0.40 \mathrm{~s} & 7.8 \pm 0.55 \mathrm{~s} \\ & 5 \mathrm{wt} \% & 6.3 \pm 1.9 \mathrm{~s} & 7.8 \pm 2.3 \mathrm{~s} & 8.0 \pm 2.4 \mathrm{~s} \\ \text { 1-HMM16A } & 2 \mathrm{wt} \% & 5.9 \pm 0.58 \mathrm{~s} & 7.6 \pm 0.69 \mathrm{~s} & 8.0 \pm 0.56 \mathrm{~s} \\ & 5 \mathrm{wt} \% & 10 \pm 1.1 \mathrm{~s} & 12 \pm 0.80 \mathrm{~s} & 12 \pm 2.0 \mathrm{~s} \\ \text { 2-HMM16A } & 2 \mathrm{wt} \% & 6.8 \pm 0.48 \mathrm{~s} & 8.6 \pm 0.60 \mathrm{~s} & 8.8 \pm 0.44 \mathrm{~s} \\ & 5 \mathrm{wt} \% & 5.6 \pm 0.17 \mathrm{~s} & 7.8 \pm 0.46 \mathrm{~s} & 7.6 \pm 0.91 \mathrm{~s} \\ & 2 \mathrm{wt} \% & 6.0 \pm 0.24 \mathrm{~s} & 7.6 \pm 0.23 \mathrm{~s} & 7.9 \pm 0.24 \mathrm{~s} \\ & 5 \mathrm{wt} \% & 8.2 \pm 1.1 \mathrm{~s} & 10 \pm 0.83 \mathrm{~s} & 9.8 \pm 0.45 \mathrm{~s}\end{array}$

${ }^{a}$ Values in columns are the degrees of recovery. ${ }^{\mathrm{b}} \mathrm{X}$ indicates no or very slow recovery after cessation of destructive strain.

\section{Conclusions}

This study has correlated structural parameters at the molecular scale of gels made from a series of highly efficient molecular gelators, m-HMMnA, whose structures are based on the selective combination of hydroxymethyl and $n$-alkyl chains around an amide group. (i.e., at the sub-nanometer distances), with those obtained at the micron and larger length scales. Furthermore, the latter have been related to mechanical properties of the systems on the macro scale and interpreted, again, based on structural data at the molecular scale. As such, we expect that the observations and conclusions derived here will be useful to those interested in the a priori design of new gelators or other molecules undergoing a variety of self-assembly processes, especially when the materials of interest are desired to be thermally and mechanically reversible.

All of the m-HMMnA gelated several alkanes and aromatic liquids, and some of the m-HMMnA formed gels in a diverse range of other liquids. The three LMOGs with 
longest alkyl chain, the m-HMM16A (i.e., with the greatest van der Waals interactions), were the most efficient gelators based upon the range of liquid types gelated and the $C G C$ and $T_{g e l}$ values of their gels. At the same alkyl chain length, the LMOGs with two hydroxymethyl groups, the three 2-HMMnA, were able to gelate more liquids than the 1- or 3-HMMnA analogues. From these results and other aspects of the systems, we hypothesize that when intra-molecular H-bonding interactions become much stronger than the inter-molecular ones, gelation becomes much more dependent on amide H-bonding and van der Waals energies. Under those circumstances, the LMOGs aggregate into objects that are less likely to form strong SAFIN assemblies.

At equal concentrations of an m-HMM12A or m-HMM16A gelator, the $T_{\text {gel }}$ transition temperatures were significantly higher for gels with an $n$-alkane than with an aromatic liquid. Also, $T_{\text {gel }}$ increased as the alkyl chain length of the gelator increased. These trends cannot be attributed to differences among the $C G C$ values because they are comparable in all of these gels; the amounts of gelator contributing to the SAFINs are almost the same. In fact, the different behaviors must be due to the types of gelator-liquid interactions being assessed by the Hansen solubility parameters at the different stages of aggregation, nucleation, and growth of the objects responsible for the SAFINs or the micro/macro phase-separated species not leading to gels.

Another manifestation of these interactions is seen in the branching patterns of the SAFINs. They depend as well on the cooling protocol for transforming the sols to gels and on the $T_{g e l}$ values, both of which determine the thermodynamic driving force for nucleation and growth of the molecules into SAFINs. Generally, at relatively low degrees of super-cooling (i.e., temperatures slightly below $T_{g e l}$ ), the gelator molecules in the sols aggregate and nucleate into fibers with less branching, because the growth of the $0 \mathrm{D}$ and $1 \mathrm{D}$ objects is more discriminating. ${ }^{3,4}$ Under conditions of greater super-cooling (i.e., temperatures much lower than $T_{g e l}$ ), aggregation, nucleation, and SAFIN growth rates are accelerated, leading to less selectivity in the processes, and 
more branching of fibers (or spherulite formation).

Perhaps most importantly, gels formed in nitrobenzene (a liquid selected for extensive study because of its low volatility and efficiency in gelating many of the m-HMMnA), exhibited partial or nearly complete viscoelastic recovery after the cessation of destructive mechanical strain. In many of the gels, the recovery rates for viscoelasticity were very fast (in the range of 5 to $12 \mathrm{~s}$ ), and the amount of destructive strain applied had little effect on the degree of recovery. .

Although the concentrations of m-HMM10A or m-HMM16A in their nitrobenzene gels had little effect on the degree of recovery of their mechanically disturbed gels, a very different behavior was found for the nitrobenzene gels of the m-HMM12A, where increasing gelator concentration resulted in decreased recovery. This seemingly bizarre observation and a few others appearing in the recent literature, ${ }^{42}$ have led us to hypothesize that stiffer (more crystalline) SAFINs are less able to recover their networks than are more elastic (less crystalline) ones. Regardless, the very fast recovery times indicate that the SAFINs are not completely destroyed by high shear/stress. It follows that (1) the constituent $1 \mathrm{D}$ objects must be retained in large part and (2) the hydroxyl groups at their surfaces must allow facile and relatively indiscriminate reassembly into the 3D SAFINs. ${ }^{27}$ These findings, when compared with comportment of the large majority of other molecular gels showing significant degrees of viscoelastic recovery, indicate that the internal and surface structures of the fibrillar objects in the m-HMMnA SAFINs are the source of the very short recovery times and high degrees of recovery after the cessation of mechanically-applied destructive force. ${ }^{42}$ It will be interesting to test this hypothesis in the future with gels comprised of a variety of structurally different gelators than those employed here in which the number and location of functional groups responsible for the mechano-reversibility are varied. Already, the gels reported here may find applications in lubrication and drug delivery. 


\section{AUTHOR INFORMATION}

\section{Corresponding Author}

E-mail: weissr@georgetown.edu.

\section{Acknowledgments}

YZ is grateful to the China Scholarship Council (CSC) for a fellowship to support her study at Georgetown University. We thank Drs. Mohan Zhang, Jingjing Li, V. Ajay Mallia and Xinran Zhang for their help with some data collection and useful discussions. RGW thanks the US National Science Foundation for its support of this research through grant CHE-1502856.

\section{References}

1. Babu, S. S.; Praveen, V. K.; Ajayaghosh. A. Functional $\pi$-gelators and their applications. Chem. Rev. 2014, 114, 1973-2129.

2. Abdallah, D. J.; Weiss, R. G. n-Alkanes gel $n$-alkanes (and many other organic liquids). Langmuir 2000, 16, 352-355.

3. Weiss, R. G.; Terech, P. (Eds.) Molecular Gels: Materials with Self-Assembled Fibrillar Networks; Springer: Dordrecht, The Netherlands, 2006.

4. Terech, P.; Weiss, R. G. Low molecular mass gelators of organic liquids and the properties of their gels. Chem. Rev. 1997, 97, 3133-3159.

5. Amanokura, N.; Yoza, K.; Shinmori, H.; Shinkai, S.; Reinhoudt, D. N. New sugar-based gelators bearing a $p$-nitrophenyl chromophore: remarkably large 
influence of a sugar structure on the gelation ability. J. Chem. Soc. Perkin Trans. 2 1998, 2585-2592.

6. Hanabusa, K.; Maesaka, Y.; Kimura, M.; Shirai. H. New gelators based on 2-amino-2-phenylethanol: Close gelator-chiral structure relationship. Tetrahedron Lett. 1999, 40, 2385-2388.

7. Sangeetha, N. M.; Maitra, U. Supramolecular gels: functions and uses. Chem. Soc. Rev. 2005, 34, 821-836.

8. Jonkheijm, P.; van der Schoot, P.; Schenning, A. P.; Meijer, E. Probing the solvent-assisted nucleation pathway in chemical self-assembly. Science 2006, $313,80-83$.

9. Morales, M.; Gallardo, V.; Clarés, B.; García, M.; Ruiz, M. Study and description of hydrogels and organogels as vehicles for cosmetic active ingredients. $J$. Cosmet. Sci. 2009, 60, 314-314(1).

10. Vintiloiu, A.; Leroux, J. C. Organogels and their use in drug delivery-a review. J. Control Release 2008, 125, 179-192.

11. Toro-Vazquez, J. F.; Morales-Rueda, J. A.; Dibildox-Alvarado, E.; Charó-Alonso, M. A.; Alonzo-Macías, M.; González-Chávez, M. M. Development of organogels with candelilla wax and safflower oil with high triolein content. J. Am. Oil. Chem. Soc. 2007, 84, 989-1000.

12. Sijbesma, R. P.; Beijer, F. H.; Brunsveld, L.; Folmer, B. J.; Hirschberg, J. H.; Lange, R. F. Reversible polymers formed from self-complementary monomers using quadruple hydrogen bonding. Science 1997, 278, 1601-1604.

13. Cordier, P.; Tournilhac, F.; Soulié-Ziakovic, C.; Leibler, L. Self-healing and thermoreversible rubber from supramolecular assembly. Nature 2008, 451, 977-980.

14. Burattini, S.; Greenland, B. W.; Merino, D. H. A healable supramolecular 
polymer blend based on aromatic $\pi-\pi$ stacking and hydrogen-bonding interactions. J. Am. Chem. Soc. 2010, 132, 12051-12058.

15. Weng, W.; Beck, J. B.; Jamieson, A. M.; Rowan, S. J. Understanding the mechanism of gelation and stimuli-responsive nature of a class of metallo-supramolecular gels. J. Am. Chem. Soc. 2006, 128, 11663-11672.

16. Hamilton, T. D.; Bučar, D.; Baltrusaitis, J.; Flanagan, D. R.; Li, Y. J.; Ghorai, S.; Tivanski, A. V.; MacGillivray, L. R. Thixotropic hydrogel derived from a product of an organic solid-state synthesis: properties and densities of metalorganic nanoparticles. J. Am. Chem. Soc. 2011, 133, 3365-3371.

17. Huang, X.; Terech, P.; Raghavan, S. R.; Weiss, R. G. Kinetics of $5 \alpha$-cholestan-3 $\beta$-yl $N$-(2-naphthyl) carbamate/n-alkane organogel formation and its influence on the fibrillar networks. J. Am. Chem. Soc. 2005, 127, 4336-4344.

18. Huang, X.; Raghavan, S. R.; Terech, P.; Weiss, R. G. Distinct kinetic pathways generate organogel networks with contrasting fractality and thixotropic properties. J. Am. Chem. Soc. 2006, 128, 15341-15352.

19. Xue, M.; Gao, D.; Liu, K.; Peng, J.; Fang, Y. Cholesteryl derivatives as phase-selective gelators at room temperature. Tetrahedron 2009, 65, 3369-3377.

20. Xiao, H.; Srinivas, R.; Pierre, T.; Richard, G. W. Distinct kinetic pathways generate organogel networks with contrasting fractality and thixotropic properties. J. Am. Chem. Soc. 2006, 128, 15341-15352.

21. Michihiro, S.; Norifumi, F.; Seiji, S. A stable single piece of unimolecularly $\pi$-stacked porphyrin aggregate in a thixotropic low molecular weight gel: a one-dimensional molecular template for polydiacetylene wiring up to several tens of micrometers in length. J. Am. Chem. Soc. 2005, 127, 4164-4165.

22. Pritam, M.; Norifumi, F.; Akihiko, T.; Takanori, K.; Michihiro, S.; Seiji, S. Regulation of a real-time self-healing process in organogel tissues by molecular adhesives. Angew. Chem. Int. Edit. 2010, 49, 6338-6342. 
23. Lescanne, M.; Grondin, P.; d'Aléo, A.; Fages, F.; Pozzo, J.-L.; Monval, O. M.; Reinheimer, P.; Colin. A. Thixotropic organogels based on a simple $N$-hydroxyalkyl amide: Rheological and aging properties. Langmuir 2004, 20, 3032-3041.

24. Brinksma, J.; Feringa, B. L.; Kellogg, R. M.; Vreeker, R.; Esch, J. V. Rheology and thermotropic properties of bis-urea-based organogels in various primary alcohols. Langmuir 2000, 16, 9249-9255.

25. Toro-Vazquez, J. F.; Morales-Rueda, J.; Torres-Martínez, A.;Charó-Alonso, M. A.; Mallia, V. A.; Weiss, R. G. Cooling rate effects on the microstructure, solid content, and rheological properties of organogels of amides derived from stearic and (R)-12-hydroxystearic acid in vegetable oil. Langmuir 2013, 29, $7642-7654$.

26. Mallia, V. A.; George, M.; Blair, D. L.; Weiss, R. G. Robust organogels from nitrogen-containing derivatives of $(R)$-12-hydroxystearic acid as gelators: comparisons with gels from stearic acid derivatives. Langmuir 2009, 25, $8615-8625$.

27. Mallia, V. A.; Weiss, R. G. Structural bases for mechano-responsive properties in molecular gels of $(R)$-12-hydroxy- $N$-( $\omega$-hydroxyalkyl)octadecanamides. Rates of formation and responses to destructive strain. Soft Matter 2015, 11, 5010-5022.

28. Yu, X.; Cao, X.; Chen, L.; Lan, H.; Liu, B.; Yi, T. Thixotropic and self-healing triggered reversible rheology switching in a peptide-based organogel with a cross-linked nano-ring pattern. Soft Matter 2012, 8, 3329-3334.

29. Du, C.; Falini, G.; Fermani, S.; Abbott, C.; Moradian-Oldak, J. Supramolecular assembly of amelogenin nanospheres into birefringent microribbons.

Science 2005, 307, 1450-1454.

30. Strader, C. R.; Pearce, C. J.; Oberlies, N. H. Fingolimod (FTY720): A recently approved multiple sclerosis drug based on a fungal secondary metabolite. J. Nat. 
Prod. 2011, 74, 900-907.

31. Hristov, A. N.; Domitrovich, C.; Wachter, A.; Cassidy, T.; Lee, C.; Shingfield, K.J.; Kairenius, P.; Davis, J.; Brown, J. Effect of replacing solvent-extracted canola meal with high-oil traditional canola, high-oleic acid canola, or high-erucic acid rapeseed meals on rumen fermentation, digestibility, milk production, and milk fatty acid composition in lactating dairy cows. J. Dairy Sci. 2011, 94, 4057-4074.

32. Abulateefeh, S. R.; Khanfar, M. A.; Al Bakain, R. Z.; Taha, M. O. Synthesis and characterization of new derivatives of alginic acid and evaluation of their iron (III) crosslinked beads as potential controlled release matrices. Pharmaceut. Dev. Tech. 2014, 19, 856-867.

33. Bag, B. G.; Dinda, S. K.; Dey, P. P.; Mallia, V. A.; Weiss, R. G. Self-assembly of esters of arjunolic acid into fibrous networks and the properties of their organogels. Langmuir 2009, 25, 8663-8671.

34. Bag, B. G.; Dinda, S. K.; Majumdar, R.; Dey, P. P.; Maity, G. C.; Mallia, V. A.; Weiss, R. G.. Self-assembly of ketals of arjunolic acid into vesicles and fibers yielding organogels. Langmuir 2013, 29, 1766-1778.

35. Ma, K.; Somashekhar, B. S.; Nagana Gowda, G. A.; Khetrapal, C. L.; Weiss, R. G. amphotropic and thermotropic ionic liquid crystallinity in phosphonium halides: "Lubrication" by hydroxyl groups. Langmuir 2008, 24, 2746-2758.

36. Escuder, B.; LLusar, M.; Miravet, J. F. Insight on the NMR study of supramolecular gels and its application to monitor molecular recognition on self-assembled fibers. J. Org. Chem. 2006, 71, 7747-7752. 
37. Bag, B. G.; Majumdar, R. Self-assembly of arjunolic acid derivatives. J. Indian Chem. Soc. 2013, 90, 1821-1828.

38. Braja, G. B.; Dash, S. S. Hierarchical self-assembly of a renewable nanosized pentacyclic dihydroxy-triterpenoid betulin yielding flower-like architectures. Langmuir 2015, 31, 13664-13672.

39. Zhang, M.; Selvakumar, S.; Zhang, X. R.; Sibi, M. P.; Weiss, R. G. Structural and solubility parameter correlations of gelation abilities for dihydroxylated derivatives of long-chain, naturally occurring fatty acids. Chem. Eur. J. 2015. 21, 8530-8543.

40. Rogers, M. A.; Abraham, S.; Bodondics, F.; Weiss, R. G. Influence of the hydroxyl position in racemic hydroxyoctadecanoic acids on the crystallization kinetics and activation energies of gels and dispersions in mineral oil. Cryst. Grow. Des. 2012, $12,5497-5504$.

41. Saito, Y.; Okano, T.; Gaill, F.; Chanzy, H.; Putaux, J. L. Structural data on the intra-crystalline swelling of beta-chitin. Int. J. Biol. Macromol. 2000, 28, 81-88.

42. Mallia, V. A.; Weiss, R. G. Correlations between thixotropic and structural properties of molecular gels with crystalline networks. Soft Matter 2016, 12, 3665-3676.

43. Matthieu, R.; Laurent, B. Organogel formation rationalized by Hansen solubility parameters. Chem. Commum. 2011, 47, 8271-8273.

44. Gao, J.; Wu, S.; Rogers, M. Harnessing Hansen solubility parameters to predict organogel formation. J. Mater. Chem. 2012, 22, 12651-12658.

45. Diehn, K. K.; Oh, H.; Hashemipour, R.; Weiss, R. G.; Raghavan, S. R. Insights into organogelation and its kinetics from Hansen solubility parameters. Toward a priori predictions of molecular gelation. Soft Matter 2014, 10, 2632-2640.

46. Mallia, V. A.; Seo, H. I.; Weiss, R. G. Influence of anions and alkyl chain lengths of $N$-alkyl-n-(R)-12-hydroxyoctadecyl ammonium salts on their 
hydrogels and organogels. Langmuir 2013, 29, 6476-6484.

47. Takahashi, A.; Sakai, M.; Kato, T. Melting temperature of thermally reversible gel. VI. Effect of branching on the sol-gel transition of polyethylene gels. Polym. J. 1980, 12, 335-341.

48. Grulke, E. A. Solubility parameter values. John Wiley \& Sons: New York, 2005

49. Hansen, C. M. Hansen solubility parameters: a user's handbook. CRC Press: Boca Raton, 2007.

50. Masahiro, S.; Chiho, S.; Hirofusa, S.; Kenji, H. Organogelation by polymer organogelators with a $L$-lysine derivative: formation of a three-dimensional network consisting of supramolecular and conventional polymers. Chemistry 2007, 13, 8193-8200.

51. Masahiro, S.; Kenji, H. Polymer organogelators that make supramolecular organogels through physical cross-linking and self-assembly. Chem. Soc. Rev. 2010, 39, 455-463.

52. Yan, N.: Xu, Z.; Diehn, K. K.; Raghavan, S. R.; Fang, Y.; Weiss, R. G. How do liquid mixtures solubilize insoluble gelators? Self-assembly properties of pyrenyl-linker-glucono gelators in tetrahydrofuran-water mixtures. J. Am. Chem. Soc. 2013, 135, 8989-8999.

53. Gao, J.; Wu, S.; Rogers, M. Harnessing Hansen solubility parameters to predict organogel formation. J. Mater. Chem. 2012, 22, 12651-12658.

54. Raynal, M.; Bouteiller, L. Organogel formation rationalized by Hansen solubility parameters. Chem. Commun. 2011, 47, 8271-8273.

55. Lin, Y. C.; Kachar, B.; Weiss, R. G. Liquid-crystalline solvents as mechanistic probes. Novel family of gelators of organic fluids and the structure of their gels. J. Am. Chem. Soc. 1989, 111, 5542-5551. 
56. Furman, I.; Weiss, R. G. Factors influencing the formation of thermally reversible gels comprised of cholesteryl 4-(2-anthryloxy) butanoate in hexadecane, 1-octanol, or their mixtures. Langmuir 1993, 9, 2084-2088.

57. Toro-Vazquez, J. F.; Juan, M. R.; Adriana, T. M.; Charó-Alonso, M. A.; Mallia, V. A.; Weiss, R. G. Cooling rate effects on the microstructure, solid content, and rheological properties of organogels of amides derived from stearic and (r)-12-hydroxystearic acid in vegetable oil. Langmuir 2013, 29, 7642-7654.

58. Douglas, J. F. Theoretical issues relating to thermally reversible gelation by supermolecular fiber formation. Langmuir 2009, 25, 8386-8391.

59. Rogers, M. A.; Marangoni, A. G. Solvent-modulated nucleation and crystallization kinetics of 12-hydroxystearic acid: A nonisothermal approach. Langmuir 2009, 25, 8556-8566.

60. Wang, R. Y.; Liu, X. Y. Kinetic studies of spherulitic crystallization in the gelation process of low molecular-mass organic gelator. Int. J. Nanosci. 2006, 5 , 645-649.

61. Wang, R. Y.; Liu, X. Y.; Xiong, J. Y.; Li, J. L. Real-time observation of fiber network formation in molecular organogel: supersaturation-dependent microstructure and its related rheological property. J. Phys. Chem. B 2006, 110, 7275-7280.

62. Li, J. L.; Liu, X. Y. Microengineering of soft functional materials by controlling the fiber network formation. J. Phys. Chem. B 2009, 113, 15467-15472.

63. Liu, X. Y.; Sawant, P. D. Formation kinetics of fractal nanofiber networks in organogels. Appl. Phys. Lett. 2001, 79, 3518-3520.

64. Kageyama, Y.; Ikegami,T.; Hiramatsu, N.; Takeda, S.; Sugawara, T. Structure and growth behavior of centimeter-sized helical oleate assemblies formed with assistance of medium-length carboxylic acids. Soft Matter 2015, 11, 3550--3558 
65. Barnes, H. A.; Hutton, J. F.; Walters, K. An introduction to rheology. Elsevier: New York, 1989.

66. Li, J. L.; Liu, X. Y.; Wang, R. Y.; Xiong, J. Y. Architecture of a biocompatible supramolecular material by supersaturation-driven fabrication of its fiber network. J. Phys. Chem. B 2005, 109, 24231-24235.

67. Abraham, S.; Lan, Y.; Lam, R. S.; Grahame, D. A.; Kim, J. J.; Weiss, R. G.; Rogers, M, A. Influence of positional isomers on the macroscale and nanoscale architectures of aggregates of racemic hydroxyoctadecanoic acids in their molecular gel, dispersion, and solid states. Langmuir 2012, 28, 4955-4964.

68. Snyder, R. G. Vibrational spectra of crystalline n-paraffins: Part I. Methylene rocking and wagging modes. J. Mol. Spectrosc. 1960, 4, 411-434.

69. Cross, W. M.; Kellar, J. J.; Miller, J. D. Fourier transform near-infrared examination of the coagel-to-micelle transition for sodium laurate. Appl. Spectrosc. 1992, 46, 701-704.

70. Ostuni, E.; Kamaras, D. P.; Weiss.R. G. Novel X-ray method for in situ determination of gelator strand structure: polymorphism of cholesteryl anthraquinone-2-carboxylate. Angew. Chem. Int. Ed. Engl. 1996, 35, 1324-1326.

71. Barnes. H. A. Thixotropy-a review. J. Non-Newton. Fluids 1997, 70, 1-33.

72. Séréro, Y.; Jacobsen, V.; Berret, J. F.; May, R. Evidence of nonlinear chain stretching in the rheology of transient networks. Macromolecules 2000, 33, 1841-1847.

73. Gupta, P. K.; Mauro, J. C. Two factors governing fragility: stretching exponent and configurational entropy. Phys. Rev. E: Stat. Nonlin. Soft Matter Phys. 2008, 78, 3172-3177.

74. Ramos, L.; Cipelletti, L. Ultraslow dynamics and stress relaxation in the aging 
of a soft glassy system. Phys. Rev. Lett. 2002, 87, 358-358.

TOC

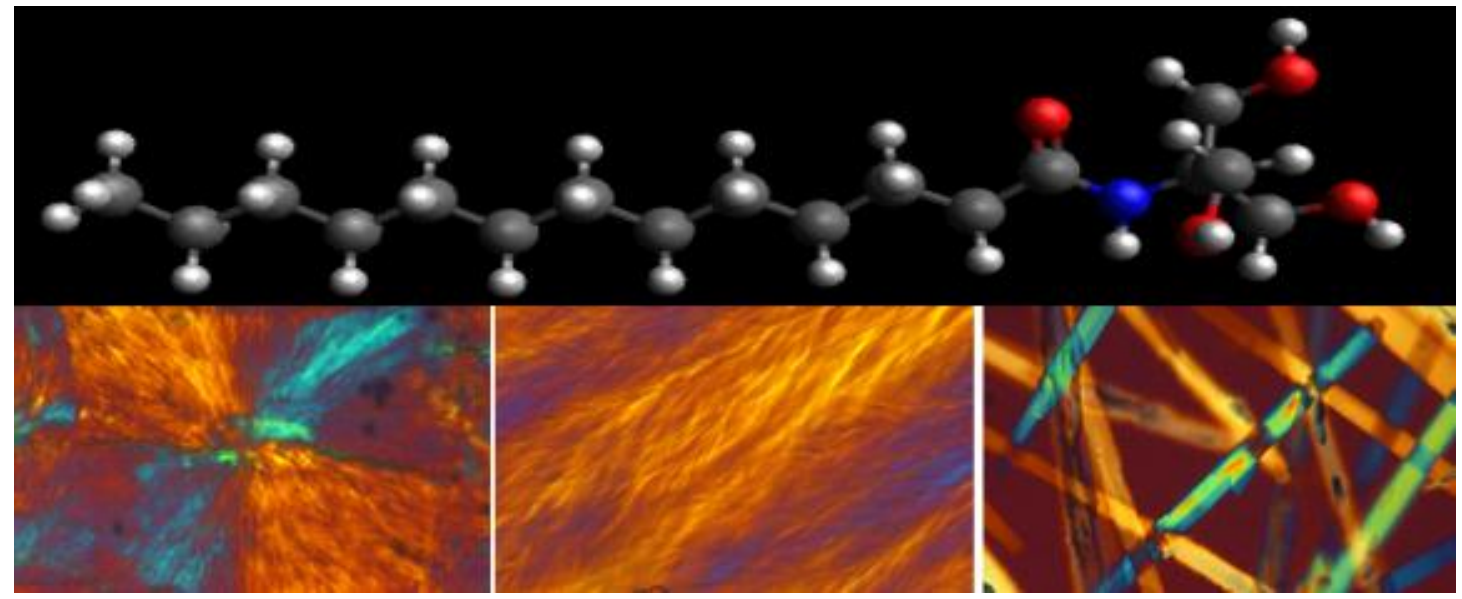

